\title{
Finite Sample Properties of Indirect Nonparametric Closed-Loop Identification
}

\author{
James S. Welsh and Graham C. Goodwin, Fellow, IEEE
}

\begin{abstract}
This paper presents new results on the properties of indirect nonparametric estimation using closed-loop data. Specific results to be developed include finite sample bias and variance. We show that previous asymptotic results hold only when the signal-to- noise ratio (SNR) is large. We develop an expression which holds generally and which departs significantly from the known asymptotic results. Simulations are presented which substantiate the validity of the general expression.
\end{abstract}

Index Terms-Bias, closed-loop identification, indirect identification, nonparametric, variance.

\section{INTRODUCTION}

C LOSED-LOOP identification has been a topic of interest for some time. This is due to many factors including the fact that many industrial processes require that data be collected under closed-loop conditions for safety or other operational reasons. Here, we will be concerned with nonparametric estimation [1]-[4]. Specifically, we consider the indirect method of identification [1], [2], [4], and [5].

The indirect method first requires that one fit a model to the closed-loop system. Then, using knowledge of the controller, one back-solves for the corresponding open-loop model of the process. This method has been well studied. However, many of the key properties, especially under finite sample conditions are, as yet, unresolved. A key difficulty turns out to be a fundamental singularity in the indirect procedure. Here, we overcome this problem by a regularization technique based on use of an exclusion zone.

Our analysis leads to general results for bias and variance. When specialized to the case of high signal-to-noise ratio (SNR), our results turn out to be consistent with earlier findings [1], [3], [4], [6]. However, a key conclusion arising from the work presented here is that the earlier approximate results are valid only when the variance is negligibly small. By way of contrast, our results hold for arbitrary SNR.

The layout of the remainder of the paper is as follows. A review of previous work on indirect nonparametric estimation is provided in Section II. In Section III, we introduce notation by briefly summarizing known results on open-loop nonparametric identification. Section IV explains the indirect estimate obtained in closed-loop, sets up the key exclusion zone idea, and details the associated assumptions. Section V gives a heuristic statement of the results to follow. Section VI provides prelimi-

Manuscript received August 11, 2000; revised May 23, 2001 and February 6, 2002. Recommended by Associate Editor J. Chen.

The authors are with the Centre for Integrated Dynamics and Control, The University of Newcastle, Callaghan, 2308 NSW, Australia (e-mail jwelsh@ee.newcastle.edu.au; eegcg@ee.newcastle.edu.au).

Publisher Item Identifier 10.1109/TAC.2002.800768.

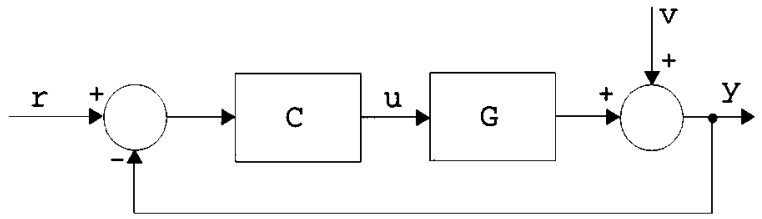

Fig. 1. Closed-loop system.

nary technical results on the probability density function for finite sample data with an exclusion zone. Sections VII and VIII give a rigorous development of the bias and variance results. Section IX examines design issues associated with the application of the bias and variance results as well as issues regarding the size of the exclusion zone. Section X presents a simulation example and Section XI draws conclusions. To facilitate readability, details of proofs are given in the Appendix.

\section{OVERVIEW OF INDIREDT NONPARAMETRIC IDENTIFICATION}

Consider the closed-loop system shown in Fig. 1. We examine the special case when the external reference signal $r$ is periodic and we assume, so as to avoid transients, that the reference has been applied for some time before samples are taken. Also, we consider a square window function. The use of other windows would not change the main thrust of our argument [7].

In open loop, the least mean square estimate of the frequency response of a linear system is given by the ratio of the input and output discrete Fourier transforms (DFTs). As the calculation of the frequency response depends upon ratios, the scaling of the DFT is irrelevant. Thus, the estimate, $\hat{T}$, of the closed-loop transfer function from $r$ to $y$ is given by

$$
\hat{T}_{i}=\frac{Y_{i}}{R_{i}}
$$

where $Y_{i}$ and $R_{i}$ denote the DFT, at frequency $\omega_{i}$, of output and reference, respectively, and $\hat{T}_{i}$ denotes the estimate of the closed-loop transfer function at frequency $\omega_{i}$.

This estimate has also been called the empirical transfer function estimate (ETFE). It is known [1] that this estimate has poor asymptotic properties when the input is a stochastic process. In fact, the variance of the ETFE for a stochastic input has been shown to be infinite [8]. However, the method is known to give good results when the input is periodic [1], [7].

The true closed-loop transfer function at frequency $\omega_{i}$, which we denote by $T_{o i}$, can be expressed as

$$
T_{o i}=\frac{G_{i} C_{i}}{1+G_{i} C_{i}}
$$

where $G_{i}$ and $C_{i}$ are the transfer functions of the process and controller, respectively, evaluated at frequency $\omega_{i}$ (see Fig. 1). 
Inverting the relationship in (2) suggests the following indirect estimate for $G$ at frequency $\omega_{i}$

$$
\hat{G}_{i}=\frac{\hat{T}_{i}}{C_{i}\left(1-\hat{T}_{i}\right)}
$$

where $\hat{T}_{i}$ is given in (1).

Our objective here is to study the properties of $\hat{G}_{i}$ as given in (3) and (1). Recent work on this problem includes [1], [5], and [9], which all build on classical methods for time series analysis in the frequency domain [10]-[14]. Various approximate results for bias and variance have been described in the literature. For example, in [6], it is argued that, when the SNR is low, then $\hat{G}_{i}$ approaches $-1 / C_{i}$. Also, in [1], [4], and [6], it has been argued that in high SNR environments the bias approaches zero and the variance is a simple function of the output noise power to input energy at the frequency of interest. A difficulty with these results is that they depend on the sample property of having high SNR which is not verifiable from the data.

Results in [15] and [16] corroborate these approximations by showing that, for high SNR, the probability density function of the estimate is concentrated around the true value and asymptotically approximates a complex Gaussian distribution. Moreover, in [9], an exact expression is given for the bias of the estimate using the Cauchy principal value to deal with the singularity at $\hat{T}_{i}=1$ inherent in (3). In a similar vein, it has also been shown in [9] that the variance is infinite due again to the singularity at $\hat{T}_{i}=1$. This result is similar to the result for open-loop systems when the input contains noise signals [8], [17], [18].

It can be seen from the previous discussion that substantial difficulties arise from the singularity of (3) at $\hat{T}_{i}=1$. Indeed, (3) is an example of an ill-conditioned inverse problem. As mentioned in Section I, our solution to this difficulty is to use regularization based on an exclusion zone. In particular, we require that, at the frequency of interest, the true closed-loop transfer function, $T_{o i}$, be bounded away from one. This allows us to discard any value of $\hat{T}_{i}$ that falls in the vicinity of one. A similar idea of using exclusion zones to deal with a potential singularity has recently been used in [17] in the context of errors in variables estimation. We utilize this idea here to develop an expression for bias and variance which takes account of the fact that neither $T_{o i}$ nor $\hat{T}_{i}$ will lie in a region surrounding the point 1 in the complex plane. The effect of the exclusion zone size on the results is also examined. A range of exclusion zone sizes is provided, to be used as a guide, when applying the bias and variance results.

A technical difficulty is that, with finite samples, the real and imaginary parts of the estimate $\hat{T}_{i}$ are correlated [19]. Asymptotically, this correlation becomes negligible. However, a rigorous analysis of finite sample properties depends on explicitly accounting for this correlation. We will show how this can be achieved in the sequel.

\section{PROPERTIES OF OPEN-LOOP NONPARAMETRIC ESTIMATION}

In this section, we summarize known results on the nonparametric estimation of linear frequency responses from open-loop data. We will build on these results in the sequel. Proofs are given for the convenience of the reader in [20].

We make the following assumptions.
1) The reference signal $r$ is periodic with period $M$, having energy at frequencies $\omega_{1}, \ldots, \omega_{p}$ and corresponding amplitude $A_{i} ; i=1, \ldots, p$.

2) The observation period $N$ is an integer multiple of $M$.

3) The noise $v$ (see Fig. 1) is a stationary stochastic process having a Gaussian probability function and spectral density $\mathcal{S}_{v}(\omega)$ at frequency $\omega$.

Lemma 1: Part 1) The finite sample properties of the Fourier transform of the noise are described by

$$
\begin{aligned}
\mathbf{E}\left\{V_{i} V_{i}^{*}\right\} & =N \Delta \mathcal{S}_{1}\left(\omega_{i}\right) \\
\mathbf{E}\left\{V_{i} V_{i}\right\} & =\Delta \mathcal{S}_{2}\left(\omega_{i}\right)
\end{aligned}
$$

where $V_{i}$ is the DFT of the noise at $\omega_{i}=2 \pi i / N$, defined by

$$
\begin{aligned}
V_{i} & =\Delta \sum_{k=0}^{N-1} v_{k} e^{-j 2 \pi i(k / N)} \\
\Delta & =\text { sampling period (s) }
\end{aligned}
$$

$\mathcal{S}_{1}\left(\omega_{i}\right)$ is the noise spectral density $\mathcal{S}_{v}\left(\omega_{i}\right)$ convolved with a sinc $^{2}$ function centered on $\omega_{i}$, i.e.,

$$
\begin{aligned}
& \mathcal{S}_{1}\left(\omega_{i}\right)=\frac{1}{2 \pi} \int_{-\pi}^{\pi} \mathcal{S}_{v}(\omega) \Gamma_{1}\left(\omega-\omega_{i}\right) d \omega \\
& \Gamma_{1}(\omega)=\Delta \sum_{k=-\infty}^{\infty} \gamma_{1}(k) e^{-j \omega k} \quad\left(\text { a sinc }{ }^{2} \text { fn. }\right) \\
& \gamma_{1}(k)= \begin{cases}\frac{N-|k|}{N}, & \text { for }|k| \leq N \\
0, & \text { otherwise. }\end{cases}
\end{aligned}
$$

Note that (10) is a triangular function and that (9) and (10) define the Fejér kernel [21]. Also, $\mathcal{S}_{2}\left(\omega_{i}\right)$ is given by

$$
\mathcal{S}_{2}\left(\omega_{i}\right)=\Delta \sum_{k=1}^{N-1} \mathcal{R}_{v}(k) f(k, i)
$$

where

$$
\begin{aligned}
\mathcal{R}_{v}(k) & =\mathbf{E}\{v(i) v(i-k)\} \\
f(k, i) & =-2\left(\cot \frac{2 \pi i}{N}+j\right) \sin \frac{2 \pi k i}{N} .
\end{aligned}
$$

Part 2) The finite sample properties of the DFT of the periodic excitation signal applied to the reference input (see Fig. 1) are described by

$$
\begin{aligned}
& R_{i} R_{i}^{*}=g_{i}^{2}+h_{i}^{2}=\left(\frac{N \Delta}{2}\right)^{2} A_{i}^{2} \\
& R_{i} R_{i}=g_{i}^{2}-h_{i}^{2}+2 j g_{i} h_{i}
\end{aligned}
$$

where $g_{i}$ and $h_{i}$ are simple functions of the deterministic input, namely

$$
\begin{aligned}
g_{i} & =\operatorname{Re}\left\{R_{i}\right\} \\
h_{i} & =\operatorname{Im}\left\{R_{i}\right\}
\end{aligned}
$$

and $A_{i}$ is the amplitude of the input at frequency $\omega_{i}$.

Remark 2: We note that, provided $\mathcal{S}_{v}(\omega)$ is smooth, then

$$
\lim _{N \rightarrow \infty} \mathcal{S}_{1}(\omega) \rightarrow \mathcal{S}_{v}(\omega)
$$

due to the sampling properties of the $\operatorname{sinc}^{2}$ function. 


\section{A. Application to Open-Loop System Identification}

We temporarily focus on the open-loop estimation problem. The open-loop analog of (1) is

$$
\hat{G}_{i}=\frac{Y_{i}}{R_{i}}
$$

Lemma 3: Let $G_{i}$ denote the true open-loop frequency response of the system at frequency $\omega_{i}$ and define $\tilde{G}_{i}=\hat{G}_{i}-G_{i}$. Then

$$
\tilde{G}_{i}=\frac{V_{i}}{R_{i}}, \quad i=1,2, \ldots, p
$$

where $V_{i}$ and $R_{i}$ denote the DFT (at $\omega_{i}$ ) of the noise and of the reference input, respectively. We then have

$$
\begin{aligned}
& \text { i) } \mathbf{E}\left\{\tilde{G}_{i}\right\}=0 \\
& \text { ii) } \mathbf{E}\left\{\tilde{G}_{i} \tilde{G}_{i}^{*}\right\}=\left[\begin{array}{cc}
\gamma_{1 i} & \gamma_{3 i} \\
\gamma_{3 i} & \gamma_{2 i}
\end{array}\right]=D
\end{aligned}
$$

where

$$
\begin{aligned}
\gamma_{1 i} & =\frac{2 \mathbf{E}\left\{V_{i} V_{i}^{*}\right\}}{(N \Delta)^{2} A_{i}^{2}}+\frac{1}{2} \operatorname{Re}\left[\frac{\mathbf{E}\left\{V_{i} V_{i}\right\}}{\left(g_{i}^{2}-h_{i}^{2}\right)+2 j g_{i} h_{i}}\right] \\
\gamma_{2 i} & =\frac{2 \mathbf{E}\left\{V_{i} V_{i}^{*}\right\}}{(N \Delta)^{2} A_{i}^{2}}-\frac{1}{2} \operatorname{Re}\left[\frac{\mathbf{E}\left\{V_{i} V_{i}\right\}}{\left(g_{i}^{2}-h_{i}^{2}\right)+2 j g_{i} h_{i}}\right] \\
\gamma_{3 i} & =\frac{1}{2} \operatorname{Im}\left[\frac{\mathbf{E}\left\{V_{i} V_{i}\right\}}{\left(g_{i}^{2}-h_{i}^{2}\right)+2 j g_{i} h_{i}}\right]
\end{aligned}
$$

and the $g_{i}, h_{i}, \mathbf{E}\left\{V_{i} V_{i}^{*}\right\}$ and $\mathbf{E}\left\{V_{i} V_{i}\right\}$ are as given in Lemma 1 .

Remark 4: We note from Lemmas 1 and 3 that for $i \neq 0$

$$
\begin{aligned}
\gamma_{1 i} & =\sigma_{i}^{2}+k_{1 i} \\
\gamma_{2 i} & =\sigma_{i}^{2}-k_{1 i} \\
\gamma_{3 i} & =k_{2 i}
\end{aligned}
$$

where

$$
\sigma_{i}^{2}=\frac{2 \mathcal{S}_{1}\left(\omega_{i}\right)}{N \Delta A_{i}^{2}}
$$

and $k_{1 i}$ and $k_{2 i}$ are of order $1 / N^{2}$. Hence, for $i \neq 0$ or $N / 2$, we have

$$
\lim _{N \rightarrow \infty} N\left[\begin{array}{cc}
\gamma_{1 i} & \gamma_{3 i} \\
\gamma_{3 i} & \gamma_{2 i}
\end{array}\right]=\left[\begin{array}{cc}
\frac{2 \mathcal{S}_{1}\left(\omega_{i}\right)}{\Delta A_{i}^{2}} & 0 \\
0 & \frac{2 \mathcal{S}_{1}\left(\omega_{i}\right)}{\Delta A_{i}^{2}}
\end{array}\right] .
$$

Note that this result holds exactly for finite $N$ in the case of a white noise source [22].

Remark 5: From Lemma 3, we see that asymptotically

$$
\frac{\mathbf{E}\left\{\tilde{G}_{i} \tilde{G}_{i}^{*}\right\}}{G_{i} G_{i}^{*}}=\frac{4 \mathcal{S}_{1}\left(\omega_{i}\right)}{\left|G_{i}\right|^{2} N \Delta A_{i}^{2}} .
$$

This has a nice physical interpretation, namely asymptotically the normalized mean square error in the frequency response estimate is two times the ratio of the output noise spectral density (windowed by a sinc $c^{2}$ function) to the output energy due to the test signal at frequency $\omega_{i}$.
In the sequel, we will focus on a single frequency, $\omega_{i}$. Thus, for clarity, we will not show the explicit dependence of $\hat{G}$, etc., on $i$.

\section{B. Application to Complementary Sensitivity Estimation}

We next consider the closed-loop system of Fig. 1. The results of Section III-A can clearly be applied to the estimation of the complementary sensitivity function, $T_{o}$, via (1). The only changes necessary to apply the results of Section III-A are as follows.

1) The noise needs to be transformed to the closed-loop output where it has spectral density

$$
\mathcal{S}_{w}(\omega)=\left|S_{o}(\omega)\right|^{2} \mathcal{S}_{v}(\omega)
$$

where

$$
S_{o}(\omega)=\frac{1}{1+G(\omega) C(\omega)} .
$$

2) The output signal energy becomes

$$
\text { output energy }=\frac{\left|T_{o}\right|^{2} N \Delta A^{2}}{2} .
$$

An immediate consequence of Lemma 3 is the following.

Corollary 6: Let $T_{o}$ denote the true complementary sensitivity at frequency $\omega$ and define $\tilde{T}=\hat{T}-T_{o}$. Then

$$
\begin{aligned}
& \text { i) } \mathbf{E}\{\tilde{T}\}=0 \\
& \text { ii) } \mathbf{E}\left\{\tilde{T} \tilde{T}^{*}\right\}=\left|S_{o}\right|^{2} D \equiv \dot{D}=\left[\begin{array}{ll}
\gamma_{1} & \hat{\gamma}_{3} \\
\dot{\gamma}_{3} & \hat{\gamma}_{2}
\end{array}\right] \\
& \text { iii) } \lim _{N \rightarrow \infty} \dot{D} \rightarrow\left[\begin{array}{cc}
\frac{2\left|S_{o}\right|^{2} \mathcal{S}_{1}}{N \Delta A^{2}} & 0 \\
0 & \frac{2\left|S_{o}\right|^{2} \mathcal{S}_{1}}{N \Delta A^{2}}
\end{array}\right]=\left[\begin{array}{cc}
\sigma^{2} & 0 \\
0 & \sigma^{2}
\end{array}\right] .
\end{aligned}
$$

Remark 7: It follows from Corollary 6 [Parts 1) and 2)], that if the noise is Gaussian then $\tilde{T}$ has a Gaussian distribution with mean and variance as given in (35) and (36). We denote this distribution by $p(\tilde{T})$.

\section{MODIFIED InDIRECT ESTIMATION OF $G$}

We essentially use the indirect estimate of $G$ described in Section II. However, we introduce the following further assumptions that allow us to remove the singularity at $\hat{T}=1$ from this estimate.

1) At frequency $\omega_{i}$, the true value of the complementary sensitivity function, namely $T_{o}$, satisfies $\left|T_{o}-1\right|>\epsilon$.

2) The true complementary sensitivity satisfies $\left|T_{o}\right|<L$.

We modify the indirect estimator by discarding any experiment in which $|\hat{T}-1|<\epsilon$. This is easily accomplished as the experiment directly yields the estimate $\hat{T}$. The removal of the singularity by excluding a zone around $\hat{T}=1$ of radius $\epsilon$ is shown in Fig. 2.

Using this exclusion zone, we will in the sequel, show that the resulting estimate of $\hat{G}$ is essentially unbiased for a good SNR and that the variance in the estimate is, for high SNR, essentially identical to that achievable with open-loop data. 


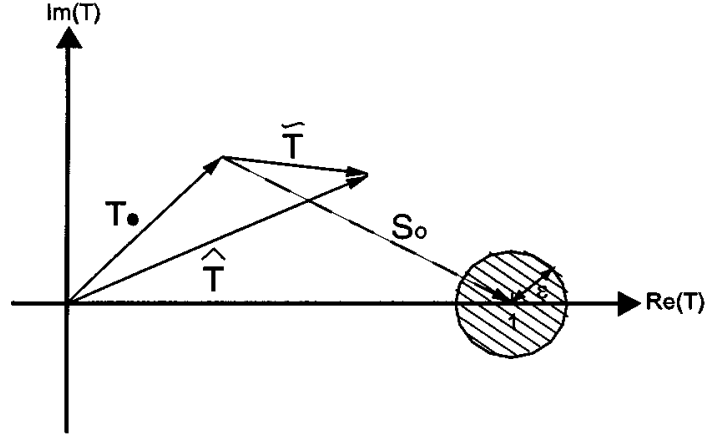

Fig. 2. Exclusion zone in $T$ space.

\section{HeUristic Statement of Bias AND VARIANCE RESUlts}

Since the subsequent derivation is rather technical, we will first give a heuristic statement of the results to be obtained so as to set the scene.

It is easily seen that the error $\tilde{G}=\hat{G}-G$ associated with the estimate (3) is given by

$$
\tilde{G}=\frac{\tilde{T}}{C\left(1-T_{o}\right)\left(1-T_{o}-\tilde{T}\right)} .
$$

Now use of the exclusion zone, ensures that $1-T_{\circ}$ and $1-T_{o}-\tilde{T}$ are bounded away from zero.

The results to be presented below hold for all SNRs. To get a feel for the results to be presented, we heuristically examine the extreme cases of low and high SNRs.

\section{A. Low SNR}

Here, $\tilde{T}$ has high variability and, thus, there is a large probability mass associated with $|\tilde{T}|>\left|1-T_{o}\right|$. These conditions suggest the following approximation to (38):

$$
\tilde{G} \simeq \frac{\tilde{T}}{C\left(1-T_{o}\right)(-\tilde{T})}=\frac{-1}{C\left(1-T_{o}\right)} .
$$

Based on this approximation, we have

$$
\begin{aligned}
\mathbf{E}\{\tilde{G}\} & \simeq \frac{-1}{C\left(1-T_{o}\right)} \\
\mathbf{E}\left\{\tilde{G} \tilde{G}^{*}\right\} & \simeq \frac{1}{|C|^{2}\left|1-T_{o}\right|^{2}}
\end{aligned}
$$

and, hence

$$
\begin{aligned}
\mathbf{E}\{\hat{G}\} & \simeq \frac{-1}{C} \\
\mathbf{E}\left\{|\hat{G}-\mathbf{E}\{\hat{G}\}|^{2}\right\} & \simeq 0 .
\end{aligned}
$$

Thus, based on this heuristic result, we might anticipate that, at low SNR, $\hat{G}$ will have mean value $-1 / C$ and very small variance around the mean.

\section{B. High SNR}

Here, $\tilde{T}$ has low variability and thus there is large probability mass associated with $|\tilde{T}|<\left|1-T_{o}\right|$. These conditions suggest the following approximation to (38):

$$
\tilde{G} \simeq \frac{\tilde{T}}{C\left(1-T_{o}\right)^{2}} .
$$

Hence, for large $N$, we would expect $\tilde{G}$ to be unbiased and

$$
\frac{\mathbf{E}\left\{\tilde{G} \tilde{G}^{*}\right\}}{G G^{*}} \simeq \frac{\mathbf{E}\left\{\tilde{T} \tilde{T}^{*}\right\}}{|G C|^{2}\left|1-T_{\circ}\right|^{4}} .
$$

Using Corollary 6, part 2), we then have

$$
\frac{\mathbf{E}\left\{\tilde{G} \tilde{G}^{*}\right\}}{G G^{*}} \simeq \frac{4\left|S_{o}\right|^{2} \mathcal{S}_{1}}{|G C|^{2}\left|S_{o}\right|^{4} N \Delta A^{2}}=\frac{4 \mathcal{S}_{1}}{\left|T_{o}\right|^{2} N \Delta A^{2}} .
$$

Thus based on this heuristic result, we might anticipate that, at high SNR, $\hat{G}$ will have mean $G$ and variance that decays as $1 / N$.

\section{Intermediate $S N R$}

It will turn out that the rigorous analysis presented later will substantiate the above heuristic expressions. In addition, our analysis will cover intermediate SNRs. To the best of our knowledge, this is the first time that these results have been obtained and are actually somewhat surprising.

\section{PREliminary TeCHNiCAL RESUlts}

Our objective in the following sections is to rigorously derive expressions (akin to those outlined in Section V) which hold in the finite sample case with an exclusion zone. The key results are given in Theorems 12, 13, and 15. As a prelude, we present here some preliminary technical results. Again, for convenience of the reader, proofs of these results can be found in [20].

We observe that since the noise is assumed zero-mean Gaussian and since $\hat{T}$ is a linear function of the data, then $\hat{T}$ and $\tilde{T}$ will also have a Gaussian distribution. Indeed, from Corollary 6 , we see that $\tilde{T}$ has a Gaussian distribution with zero mean and covariance (36).

We next express the probability density function in terms of polar coordinates via $\tilde{T}=\mathrm{Re}^{j \lambda}$. For a two-variate Gaussian random variable with zero-mean and radially symmetric covariance, the resultant probability density function is a Rayleigh distribution

$$
\not{p}(R, \lambda: \sigma)=\frac{R}{2 \pi \sigma^{2}} e^{-R^{2} / 2 \sigma^{2}} .
$$

Our goal is to essentially apply this result to $\tilde{T}$. However, a difficulty with the distribution for $\tilde{T}$ is that it is not radially symmetrical with finite sample size. To deal with this problem, we will use bounding techniques on the distribution. Essentially, this is accomplished by surrounding the elliptical distribution by inner and outer radially symmetrical distributions. Let $p_{T}(R, \lambda)$ be the transformed (true) probability density function for $\tilde{T}$ without an exclusion zone. Then

$$
p_{T}(R, \lambda)=J p(\tilde{T}) \quad \tilde{T}=\operatorname{Re}^{j \lambda}
$$

where $J$ is the Jacobian [12] linking $\tilde{T}$ to $\operatorname{Re}^{j \lambda}$. 
Lemma 8: Let $\tilde{T}=\operatorname{Re}^{j \lambda}=\operatorname{Re}[\tilde{T}]+j \operatorname{Im}[\tilde{T}]$. Then the probability density function for $\tilde{T}$ can be bounded as follows:

$$
k_{\min } \not{p}\left(R, \lambda: \sigma_{\min }\right) \leq p_{T}(R, \lambda) \leq k_{\max } \not \dot{p}\left(R, \lambda: \sigma_{\max }\right)
$$

where $\not{p}(),. p_{T}$ are as in (47) and (48), and

$$
\begin{aligned}
k_{\min } & =\frac{\sigma_{\min }^{2}}{\sqrt{\operatorname{det}(\dot{D})}} \\
k_{\max } & =\frac{\sigma_{\max }^{2}}{\sqrt{\operatorname{det}(\dot{D})}} \\
\sigma_{\min }^{2} & =\min \left(\dot{\gamma}_{1 i}, \dot{\gamma}_{2 i}\right)-\left|\dot{\gamma}_{3 i}\right| \\
\sigma_{\max }^{2} & =\max \left(\dot{\gamma}_{1 i}, \dot{\gamma}_{2 i}\right)+\left|\dot{\gamma}_{3 i}\right|
\end{aligned}
$$

and where $\dot{D}$ is given in (36).

Remark 9: In the limit as $N \rightarrow \infty$, we see for Part 3) of Corollary 6 , that $\sigma_{\max }^{2} \rightarrow \sigma^{2}$ and $\sigma_{\min }^{2} \rightarrow \sigma^{2}$. Also

$$
\frac{\sigma_{\max }^{2}}{\sqrt{\operatorname{det}(\dot{D})}} \rightarrow 1 \text { and } \frac{\sigma_{\min }^{2}}{\sqrt{\operatorname{det}(\dot{D})}} \rightarrow 1
$$

Finally, in the modified indirect estimator we remove the singularity in the estimate of $\hat{G}$ by excluding a zone around $\hat{T}=1$. In this zone, we are implicitly setting the probability to zero. This requires us to rescale the probability density function so that it integrates to unity. In the sequel, it will be convenient to recenter and scale the estimation error in the complimentary sensitivity function. Thus, we define $\tilde{T}^{\prime}=\tilde{T} / S_{o}$. In the symmetrical case, using (37), the real and imaginary parts of $\tilde{T}^{\prime}$ have variance

$$
\sigma_{s c}^{2}=\frac{\sigma^{2}}{\left|S_{o}\right|^{2}}=\frac{2 \mathcal{S}_{1}}{N \Delta A^{2}}
$$

Also, the exclusion zone, when transformed to the $\tilde{T}^{\prime}$ space remains a circle centered at $(1,0)$ but having radius $\epsilon_{1}=\epsilon /\left|S_{\circ}\right|$. We will develop expressions for renormalization constants $c_{\max }$ and $c_{\min }$ of the bounding probability density functions when the excluded zone is removed. The excluded zone is shown as the shaded area "A" in Fig. 3. We have seen (Lemma 8) that the density function $p_{T}(R, \lambda)$, although not itself a Rayleigh distribution, is bounded from above and below by scaled Rayleigh distributions. These Rayleigh density functions, with the excluded zone removed, will be expressed as

$$
\bar{p}\left(R, \lambda: \sigma_{s c}\right)=\frac{\not p\left(R, \lambda: \sigma_{s c}\right)}{c_{h}} .
$$

When $\sigma_{s c}^{2}$ is replaced by either $\sigma_{s c \text { max }}^{2}$ or $\sigma_{s c \text { min }}^{2}$ [as defined in (56) and (57)], then $c_{h}$ takes the value $c_{\max }$ or $c_{\min }$, respectively. We will quantify the constants $c_{h}, c_{\max }$ and $c_{\min }$ in the following.

Scaling of the minimum and maximum values of $\sigma_{s c}^{2}$ gives

$$
\begin{aligned}
\sigma_{s c \min }^{2} & =\frac{\sigma_{\min }^{2}}{\left|S_{o}\right|^{2}} \\
\sigma_{s c \max }^{2} & =\frac{\sigma_{\max }^{2}}{\left|S_{o}\right|^{2}} .
\end{aligned}
$$

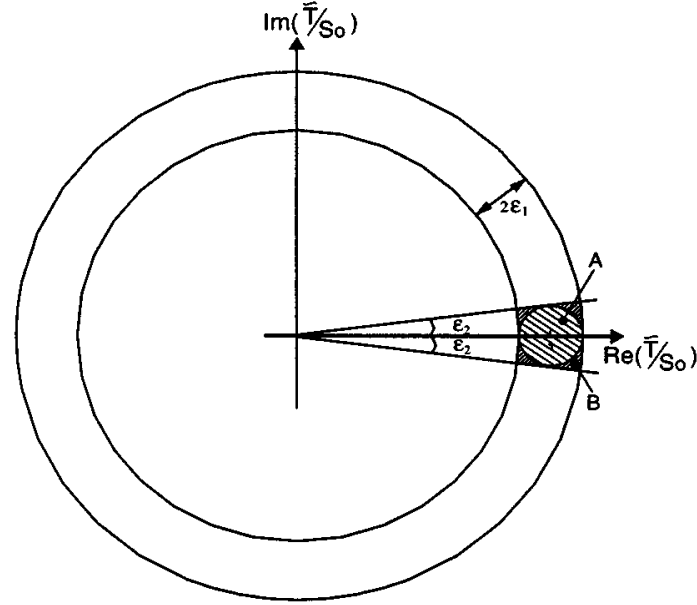

Fig. 3. Exclusion zone in $\tilde{T}$ space.

The scaled values of $k_{\min }$ and $k_{\max }$ are then given by

$$
\begin{aligned}
& k_{s c \min }=\frac{\sigma_{s c \min }^{2}}{\sqrt{\operatorname{det}(D)}} \\
& k_{s c \max }=\frac{\sigma_{s c \max }^{2}}{\sqrt{\operatorname{det}(D)}} .
\end{aligned}
$$

Lemma 10: The probability of lying outside the exclusion zone for the probability density function given in (47) is

$$
c_{h}=1-e^{-1 / 2 \sigma_{s c}^{2}} \sum_{k=0}^{\infty} \frac{1}{(k !)^{2} 2^{k} \sigma_{s c}^{2 k}} \gamma\left(k+1, \frac{\epsilon_{1}^{2}}{2 \sigma_{s c}^{2}}\right)
$$

where $\gamma\left(k+1,\left(\epsilon_{1}^{2} / 2 \sigma_{s c}^{2}\right)\right)$ is an incomplete Gamma function.

Proof of this and subsequent results are presented in the Appendix.

Remark 11: For the case of an elliptical shaped distribution, we denote the renormalized distribution as $\bar{p}_{T}(R, \lambda)$ where

$$
\bar{p}_{T}(R, \lambda)=\frac{1}{c_{1}} p_{T}(R, \lambda)
$$

and $p_{T}(R, \lambda)$ is as in (48). We will not need to explicitly evaluate $c_{1}$. Instead, we note that

$$
c_{\min } \leq c_{1} \leq c_{\max }
$$

where $c_{\max }$ and $c_{\min }$ are as in (60) with the appropriate substitutions for $\sigma_{s c}^{2}$ (i.e., $\sigma_{s c \max }^{2}, \sigma_{s c \text { min }}^{2}$ ).

\section{FInITE SAMPLE BiaS}

In this section, we develop an expression for the bias in the modified estimator. We know from Corollary 6 that asymptotically the distribution of $\tilde{T}$ becomes symmetrical. Thus we first determine an expression for the bias corresponding to the asymptotic symmetrical distribution. Then we bound the error between the true bias under finite sampling and that of the bias for the asymptotic distribution.

Theorem 12: Part 1) For the asymptotic case, when the distribution for $\tilde{T}$ has radially symmetrical covariance as in 
Part 3) of Corollary 6, then the bias in the indirect estimate of the process transfer function is

$$
\begin{array}{r}
\mathbf{E}\left\{\tilde{G}_{h}\right\}=\frac{-1}{C S_{o}}+\frac{1}{c_{h} C S_{o}}\left(1-e^{\left(-\left(1-\epsilon_{1}\right)^{2}\right) / 2 \sigma_{s c}^{2}}\right) \\
+K_{b 1}+K_{b 2}
\end{array}
$$

where, $\sigma_{s c}^{2}$ is defined in (54), $c_{h}$ is as in (60), and

$$
\begin{aligned}
K_{b 1} & =\int_{1-\epsilon_{1}}^{1+\epsilon_{1}} \int_{\epsilon_{2}}^{2 \pi-\epsilon_{2}} f_{b}(R, \lambda) d \lambda d R \\
K_{b 2} & =\iint_{B} f_{b}(R, \lambda) d \lambda d R \\
f_{b}(R, \lambda) & =\frac{\bar{p}\left(R, \lambda: \sigma_{s c}\right)}{C S_{o}\left(1-\mathrm{Re}^{j \lambda}\right)} \\
\bar{p}\left(R, \lambda: \sigma_{s c}\right) & =\left\{\begin{array}{lc}
\frac{R}{c_{h} 2 \pi \sigma_{s c}^{2}} e^{-R^{2} / 2 \sigma_{s c}^{2},} & \text { for }(R, \lambda) \\
0, & \in(\mathbb{C}-A) \\
0 & \text { for }(R, \lambda) \in A .
\end{array}\right.
\end{aligned}
$$

$$
\begin{aligned}
& A=\text { Area "A" in Fig. } 3 \\
& B=\text { Area " } \mathrm{B} \text { " in Fig. } 3
\end{aligned}
$$

Part 2) Under the conditions of Part 1), we can tightly overbound the constants $K_{b 1}$ and $K_{b 2}$ as follows:

$$
\begin{aligned}
K_{b 1} \leq & \frac{1}{\pi c_{h} C S_{o}}\left[\log \tan \left(\frac{\cos ^{-1}\left(1-\epsilon_{1}\right)}{2}\right)\right. \\
& -\log \tan \left(\frac{\epsilon_{2}}{2}\right)+\frac{\frac{\pi}{2}-\cos ^{-1}\left(1-\epsilon_{1}\right)}{\sqrt{2 \epsilon_{1}-\epsilon_{1}^{2}}} \\
& \left.+\frac{\pi}{2 \sqrt{2-2 \epsilon_{1}+\epsilon_{1}^{2}}}\right]\left(e^{\left(-\left(1-\epsilon_{1}\right)^{2}\right) / 2 \sigma_{s c}^{2}}\right. \\
K_{b 2} \leq & \frac{1}{2 \pi c_{h} \sigma_{s c}^{2} C S_{o}}\left(\left[2 \epsilon_{2}-\epsilon_{1}\left(\epsilon_{2}-\frac{\pi}{2}\right)\right]\right. \\
& \left.\cdot e^{\left(-\left(1-\epsilon_{1}\right)^{2}\right) / 2 \sigma_{s c}^{2}}+\left(1+\epsilon_{1}\right) / 2 \sigma_{s c}^{2}\right) \\
& \left.\cdot\left(2 \epsilon_{2}+\epsilon_{1}\left(\epsilon_{2}-\frac{\pi}{2}\right)\right) e^{-1 / 2 \sigma_{s c}^{2}}\right) .
\end{aligned}
$$

We next consider the finite data case when the distribution for $\tilde{T}$ is not radially symmetric. This significantly complicates the result. Thus, we will relate the bias in this case to the result developed above for the radially symmetric case. Specifically, we have the following.

Theorem 13: In a closed-loop indirect identification experiment, the bias $\mathbf{E}\{\tilde{G}\}$ in the case of an asymmetric distribution is related to the bias in the asymptotic radially symmetric case, $\mathbf{E}\left\{\tilde{G}_{h}\right\}$, as follows:

$$
\begin{aligned}
\left|\mathbf{E}\{\tilde{G}\}-\mathbf{E}\left\{\tilde{G}_{h}\right\}\right| & \leq K_{e} \int_{0}^{1-\epsilon_{1}}\left(1+R+R^{2}+\cdots\right) P_{B} d R+K_{e} \int_{1+\epsilon_{1}}^{\infty} \\
& \cdot\left(\frac{1}{R}+\frac{1}{R^{2}}+\frac{1}{R^{3}} \cdots\right) P_{B} d R+K_{e 1}+K_{e 2}
\end{aligned}
$$

where

$$
\begin{aligned}
K_{e} & =\frac{1}{|C|\left|S_{o}\right|} \\
P_{B} & =k_{s c \max } \bar{p}_{\max }(R)-k_{s c \min } \bar{p}_{\min }(R) \\
\bar{p}_{\min }(R) & =\frac{R}{c_{\min } \sigma_{s c \min }^{2}} e^{-R^{2} / 2 \sigma_{s c \text { min }}^{2}} \\
\bar{p}_{\max }(R) & =\frac{R}{c_{\max } \sigma_{s c \max }^{2}} e^{-R^{2} / 2 \sigma_{s c \max }^{2}}
\end{aligned}
$$

and $\sigma_{s c \min }^{2}, \sigma_{s c \max }^{2}, k_{s c \min }$, and $k_{s c \max }$ are defined in (56)-(59), respectively

$$
\begin{aligned}
K_{e 1} \leq & \frac{K_{e}}{\pi}\left(\log \tan \left(\frac{\cos ^{-1}\left(1-\epsilon_{1}\right)}{2}\right)-\log \tan \left(\frac{\epsilon_{2}}{2}\right)\right. \\
& +\frac{\frac{\pi}{2}-\cos ^{-1}\left(1-\epsilon_{1}\right)}{\left.\sqrt{2 \epsilon_{1}-\epsilon_{1}^{2}}+\frac{\pi}{2 \sqrt{2-2 \epsilon_{1}+\epsilon_{1}^{2}}}\right)} \\
& \cdot\left(\frac{k_{s c \max }}{c_{\max }}\left(\exp \left(\frac{-\left(1-\epsilon_{1}\right)^{2}}{2 \sigma_{s c \max }^{2}}\right)-\exp \left(\frac{-\left(1+\epsilon_{1}\right)^{2}}{2 \sigma_{s c \max }^{2}}\right)\right)\right. \\
& \left.-\frac{k_{s c \min }}{c_{\min }}\left(\exp \left(\frac{-\left(1-\epsilon_{1}\right)^{2}}{2 \sigma_{s c \min }^{2}}\right)-\exp \left(\frac{-\left(1+\epsilon_{1}\right)^{2}}{2 \sigma_{s c \min }^{2}}\right)\right)\right)
\end{aligned}
$$

$$
\begin{aligned}
K_{e 2} \leq & \frac{K_{e}}{2 \pi \sqrt{\operatorname{det}(D)}}\left(\left[2 \epsilon_{2}-\epsilon_{1}\left(\epsilon_{2}-\frac{\pi}{2}\right)\right]\right. \\
& {\left[\frac{1}{c_{\max }} \exp \left(\frac{-\left(1-\epsilon_{1}\right)^{2}}{2 \sigma_{s c \max }^{2}}\right)-\frac{1}{c_{\min }} \exp \left(\frac{-1}{2 \sigma_{s c \min }^{2}}\right)\right] } \\
+ & {\left[1+\epsilon_{1}\right]\left[2 \epsilon_{2}+\epsilon_{1}\left(\epsilon_{2}-\frac{\pi}{2}\right)\right] } \\
& \left.\cdot\left[\frac{1}{c_{\max }} \exp \left(\frac{-1}{2 \sigma_{s c \max }^{2}}\right)-\frac{1}{c_{\min }} \exp \left(\frac{-\left(1+\epsilon_{1}\right)^{2}}{2 \sigma_{s c \min }^{2}}\right)\right]\right)
\end{aligned}
$$

where $c_{\max }$ and $c_{\min }$ are as in (60) with the appropriate substitutions for $\sigma_{s c}^{2}$.

\section{A. Discussion}

Note that the true bias $\mathbf{E}\{\tilde{G}\}$ is displaced from $\mathbf{E}\left\{\tilde{G}_{h}\right\}$ both in magnitude and phase but rapidly approaches the asymptotic bias, $\mathbf{E}\left\{\tilde{G}_{h}\right\}$. Hence, for all practical purposes it suffices to use the simpler expression $\mathbf{E}\left\{\tilde{G}_{h}\right\}$ given in Theorem 12 .

It is also evident from (63) that the estimate with symmetric distribution essentially lies on a straight line between $G$, the true frequency response, and $-1 / C$. The amount the estimate is displaced from this line is due to the computable terms $K_{b 1}$ and $K_{b 2}$ which are dependent on the size of the chosen exclusion zone around the singularity, and hence can be made arbitrarily small. The bias result is essentially as presented in [15], save for modifications necessary to account for the exclusion zone.

We can rewrite the bias expression (for the symmetric case) in the following informative fashion:

$$
\begin{aligned}
\mathbf{E}\left\{\hat{G}_{h}\right\} & =\mathbf{E}\left\{G+\tilde{G}_{h}\right\} \\
& =\beta G+(1-\beta)\left(\frac{-1}{C}\right)+K_{b 1}+K_{b 2}
\end{aligned}
$$


where

$$
\begin{aligned}
& K=\frac{\left(1-\epsilon_{1}\right)^{2}}{2 N \sigma_{s c}^{2}} \\
& \beta=\frac{\left(1-e^{-K N}\right)}{c_{h}} .
\end{aligned}
$$

We also see from (63) that a rapid bifurcation occurs between an unbiased and a biased estimate depending on the SNR as captured by $\sigma_{s c}^{2}$. The asymptotic situation is summarized in the following.

Corollary 14: Part 1) The estimate is asymptotically unbiased, specifically

$$
\lim _{N \rightarrow \infty}|\mathbf{E}\{\tilde{G}\}|=0
$$

Part 2) The rate at which the bias converges to zero is of exponential order

$$
\lim _{N \rightarrow \infty}|\mathbf{E}\{\tilde{G}\}|=\bigcirc\left(e^{-N}\right)
$$

\section{Finite SAmple VARIANCE}

Here, we develop an expression for the variance of the estimator.

The skewed distribution caused by the finite samples is overbounded using the symmetric probability density function as in (49) to obtain our result.

Theorem 15: Part 1) In a closed-loop indirect identification experiment, the variance of the estimation error in the estimate of the process can be bounded by the following series expansion:

$$
\begin{aligned}
\mathbf{E}\left\{\tilde{G} \tilde{G}^{*}\right\} \leq & K_{v} \int_{0}^{1-\epsilon_{1}} R^{2}\left(1+R^{2}+R^{4}+\cdots\right) \bar{p}_{\max }(R) d R \\
& +K_{v} \int_{1+\epsilon_{1}}^{\infty}\left(1+\frac{1}{R^{2}}+\frac{1}{R^{4}}+\cdots\right) \cdot \bar{p}_{\max }(R) d R \\
& +K_{v 1}+K_{v 2}
\end{aligned}
$$

where $\bar{p}_{\max }(R)$ is as in (74)

$$
\begin{aligned}
K_{v} & =\frac{k_{s c \max }}{|C|^{2}\left|S_{o}\right|^{2}} \\
K_{v 1} & =\int_{1-\epsilon_{1}}^{1+\epsilon_{1}} \int_{\epsilon_{2}}^{2 \pi-\epsilon_{2}} f_{v}(R, \lambda) d \lambda d R \\
K_{v 2} & =\iint_{B} f_{v}(R, \lambda) d \lambda d R \\
f_{v}(R, \lambda) & =\frac{R^{2} k_{s c \max } \bar{p}\left(R, \lambda: \sigma_{s c \max }\right)}{|C|^{2}\left|S_{o}\right|^{2}\left|1-\mathrm{Re}^{j \lambda}\right|^{2}}
\end{aligned}
$$

and $\sigma_{s c}^{2}$ is given in (54).

Part 2) The integrals $K_{v 1}$ and $K_{v 2}$ can be tightly overbounded by

$$
\begin{aligned}
K_{v 1} \leq & \frac{K_{v}}{\pi c_{\max }}\left(\cot \left(\epsilon_{2}\right)-\frac{1-\epsilon_{1}}{\sqrt{2 \epsilon_{1}-\epsilon_{1}^{2}}}\right. \\
& \left.+\frac{\frac{\pi}{2}-\cos ^{-1}\left(1-\epsilon_{1}\right)}{\epsilon_{1}\left(2-\epsilon_{1}\right)}+\frac{\pi}{2\left(2-2 \epsilon_{1}+\epsilon_{1}^{2}\right)}\right) K_{3}
\end{aligned}
$$

where

$$
\begin{aligned}
K_{3}= & 2 \sigma_{s c \max }^{2}\left[\left(\frac{\left(1-\epsilon_{1}\right)^{2}}{2 \sigma_{s c \max }^{2}}+1\right) \exp \left(\frac{-\left(1-\epsilon_{1}\right)^{2}}{2 \sigma_{s c \max }^{2}}\right)\right. \\
& \left.-\left(\frac{\left(1+\epsilon_{1}\right)^{2}}{2 \sigma_{s c \max }^{2}}+1\right) \exp \left(\frac{-\left(1+\epsilon_{1}\right)^{2}}{2 \sigma_{s c \max }^{2}}\right)\right] \\
K_{v 2} \leq & \frac{K_{v}}{2 \pi c_{\max } \epsilon_{1} \sigma_{s c \max }^{2}}\left(\left[2 \epsilon_{2}-\epsilon_{1} \epsilon_{2}-\frac{\pi}{2} \epsilon_{1}\right]\right. \\
& \cdot \exp \left(\frac{-\left(1-\epsilon_{1}\right)^{2}}{2 \sigma_{s c \max }^{2}}\right)+\left[2 \epsilon_{2}+\epsilon_{1} \epsilon_{2}-\frac{\pi}{2} \epsilon_{1}\right] \\
& \left.\cdot\left[1+\epsilon_{1}\right]^{3} \exp \left(\frac{-1}{2 \sigma_{s c \max }^{2}}\right)\right) .
\end{aligned}
$$

\section{A. Discussion}

The above result gives the variance around the true value $G$. Using $\mathbf{E}\{\hat{G}\}=G_{b}$, the variance around the mean is readily
seen to be

$$
\mathbf{E}\left\{\left|\hat{G}-G_{b}\right|^{2}\right\}=\mathbf{E}\left\{|\hat{G}-G|^{2}\right\}-\left|G_{b}-G\right|^{2}
$$

where $\mathbf{E}\{\hat{G}\}=G_{b}$.

Armed with these rigorous results, it is convenient to revisit the heuristic results foreshadowed in Section V.

1) Low SNR: Under these conditions, the variance expression (83) is dominated by the first term in the second integral which gives

$$
\mathbf{E}\left\{\tilde{G} \tilde{G}^{*}\right\} \simeq \frac{1}{|C|^{2}\left|S_{o}\right|^{2}}
$$

Note that this is as foreshadowed in (41) and (43).

2) High SNR: Under these conditions, the variance expression (83) is dominated by the first term of the first integral. It is shown in the Appendix that, as the number of observations $(N)$ tends to infinity the normalized variance of the estimation error converges to a value of two times the ratio of the noise spectral density to the output energy due to the test signal at frequency $\omega_{i}$. Specifically, the following result holds:

$$
\lim _{N \rightarrow \infty}\left|\frac{\mathbf{E}\left\{N \tilde{G} \tilde{G}^{*}\right\}}{G G^{*}}-\frac{4 \mathcal{S}_{1}}{\left|T_{o}\right|^{2} \Delta A^{2}}\right|=0 .
$$

Note that the aforementioned result is the same as the low-order approximations for the variance given in [6] and [3] and as heuristically described in Section V.

An interesting observation arising from this result is that $\left|T_{o}\right|^{2} N \Delta A^{2} / 2$ is actually the output signal energy due to the test signal. Hence, if one fixes the output energy due to the test signal, then we see that estimation accuracy is the same whether open or closed-loop provided the SNR is high. [Compare (93) with Remark 5].

It is also evident from (93) that, the rate of which the variance (83) converges to the ratio of the noise spectral density and the 

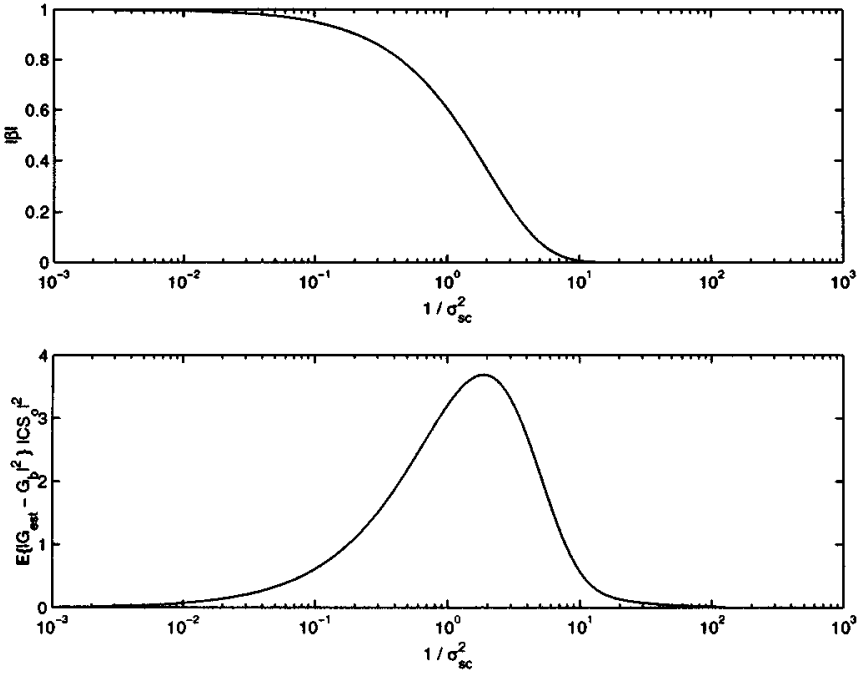

Fig. 4. (Top) Bias versus $1 / \sigma_{s c}^{2}$. (Bottom) Variance around the mean versus $1 / \sigma_{s c}^{2}$.

energy present in the output due to the excitation signal applied at the reference is of the order of $1 / N$

$$
\lim _{N \rightarrow \infty}\left|\mathbf{E}\left\{\tilde{G} \tilde{G}^{*}\right\}\right|=\bigcirc\left(\frac{1}{N}\right) .
$$

3) Intermediate SNR: To the best of our knowledge, this case has not been previously treated in the literature (even at a heuristic level). The reason is that the exclusion zone is crucial to ensuring convergence of the series in (83).

\section{DESIGN ISSUES}

In Fig. 4, we examine our results for bias and variance with respect to the SNR as captured by $1 / \sigma_{s c}^{2}$ (which is proportional to $N$, the number of observations). Here, $\epsilon$ was set to a value of 0.0035 . We see in Fig. 4(a) the rapid bifurcation occurring in the bias as foreshadowed in Section VII-A. From (80), $|\beta|=1$ is defined as biased and $|\beta|=0$ as unbiased. The heuristic and analytic results for the variance are confirmed in Fig. 4(b). As foreshadowed in Section $\mathrm{V}$, we see that the variance around the mean is zero for both high and low SNRs. An interesting observation is that the maximum of the variance occurs at a higher SNR than that of the "switching point" of the bias. Note that the results in Fig. 4 have been presented in a normalized fashion and, hence, these "design curves" can be used to calculate bias and variance for any given problem.

To compare the true variance expression with the asymptotic result given in (93), we have plotted the two expressions in Fig. 5. Note that, surprisingly, the asymptotic expression is valid only when the variance is negligibly small. Thus, it seems essential to use the general result as given in Theorem 15 .

Finally, we examine the effect the size of the exclusion zone has on our estimate of the variance around the mean with respect to the SNR. Fig. 6 shows the result of a simulation where the variance around the mean was calculated for a range of $\epsilon$ (size of exclusion zone) and $1 / \sigma_{s c}^{2}(\mathrm{SNR})$.

A preliminary observation from Fig. 6 is that one should keep $\epsilon$ away from 0 or 1 . Indeed, we see from Fig. 6, that as $\epsilon \rightarrow 0$ the

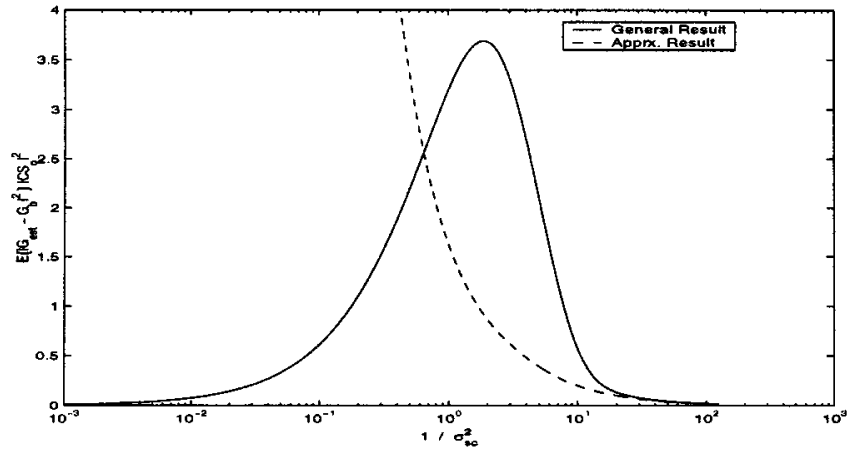

Fig. 5. Comparison of the general result and approximate result for the variance around the mean.

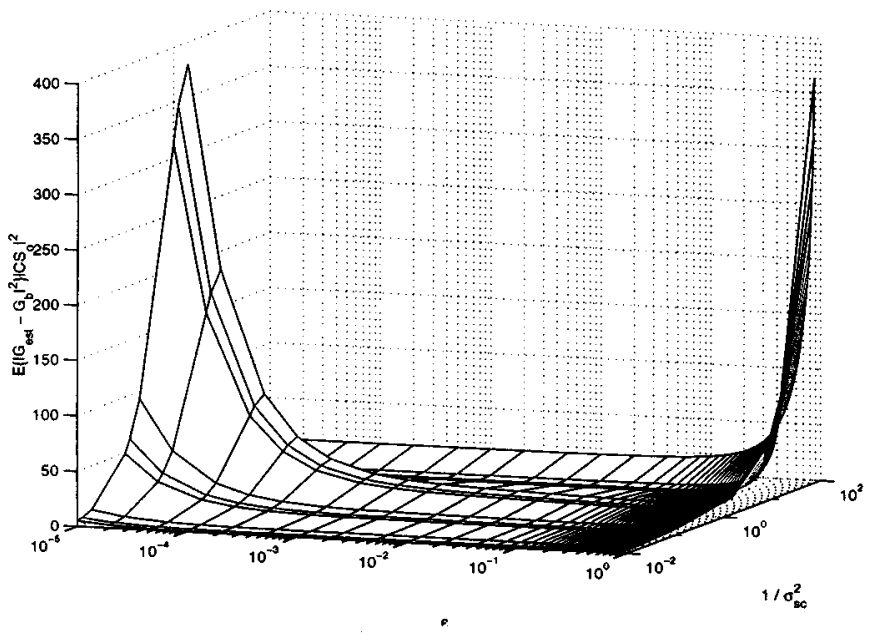

Fig. 6. Effect of exclusion zone on the variance around the mean.

variance will go to $\infty$. Of course, this is what we anticipated. Indeed, this was the original motivation for including an exclusion zone in the first place. As $\epsilon \rightarrow 1$, with a high SNR, the variance also tends toward $\infty$. This occurs since now the true system, $T_{o}$, falls within (or close to) the exclusion zone and, therefore, when the SNR is high the probability density function of the estimates is concentrated around this point and, hence, will be discarded.

Away from the values $\{0,1\}$, Fig. 6 shows the estimate of the variance is actually quite insensitive to values of $\epsilon$ between 0.005 and 0.1 for this particular value of $T_{o}$. More generally, we have found that the result is insensitive to $\epsilon$ provided the size of the exclusion zone is greater than about 0.005 and smaller then (a bound on) the distance of $T_{o}$ from 1 . Indeed, this is intuitively reasonable given the geometry of Fig. 2. This suggests that any choice of $\epsilon$ in this region will yield satisfactory results.

\section{Simulation}

To test the theory presented here, we have chosen a simple system with $G=1 /(4 s+1)$ and $C=(4 s+1) / s$.

A test signal consisting of Schroeder phased sinusoids was applied to the reference input with their amplitude made inversely proportional to their frequency. Noise was added which was normally distributed having zero mean and a spectral density of 0.1 . An experiment length of $80 \mathrm{~s}$ was used with a sampling period of $5 \mathrm{~ms}$. A Monte Carlo study was carried out utilizing 2500 tests with different noise seeds, the results of which 


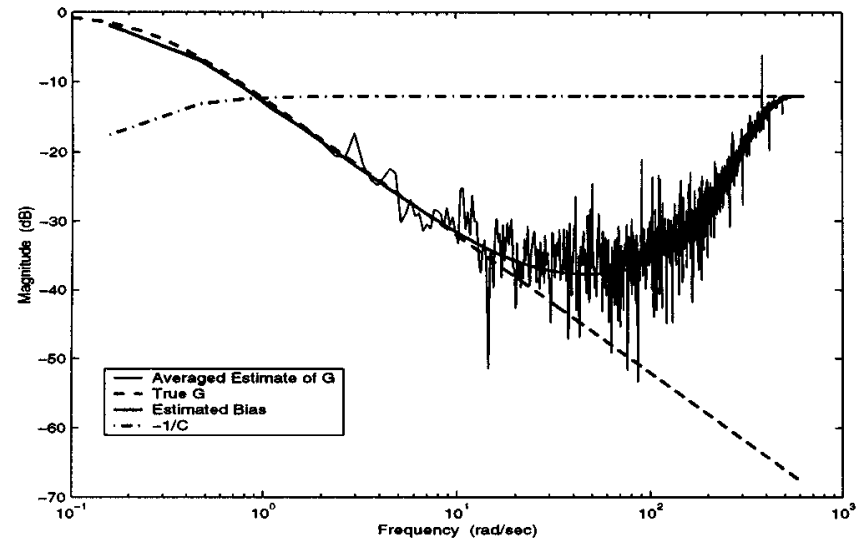

Fig. 7. Estimate of bias.

were then averaged. The bias and variance results for the averaged data are readily derived from the earlier results. Specifically, if we assume $N_{s}$ independent repetitions of the experiments and denote by $\left\{\hat{G}(k): k=1, \ldots N_{s}\right\}$, the results of each experiment, and by $\hat{G}_{a v}$ the averaged estimate, then

$$
\begin{gathered}
\hat{G}_{a v}=\frac{1}{N_{s}} \sum_{k=1}^{N_{s}} \hat{G}(k) \\
\mathbf{E}\left\{\hat{G}_{a v}\right\}=\mathbf{E}\{\hat{G}(k)\} \triangleq G_{b}
\end{gathered}
$$

and, hence

$$
\begin{aligned}
\mathbf{E}\left\{\left|\hat{G}_{a v}-G_{b}\right|^{2}\right\} & =\mathbf{E}\left\{\left|\frac{1}{N_{s}} \sum_{k=1}^{N s}\left(\hat{G}(k)-G_{b}\right)\right|^{2}\right\} \\
& =\frac{1}{N_{s}}\left[\mathbf{E}\left\{|\hat{G}(k)-G|^{2}\right\}-\left|G_{b}-G\right|^{2}\right] .
\end{aligned}
$$

The averaged estimated gains are shown in Fig. 7 together with the theoretical bias obtained using the results of Section IV. Note that Fig. 7 uses lines for the various plots whereas, in fact, only discrete frequencies were used. This was done purely for aesthetic reasons as the number of test frequencies used in the simulation was 2000, making the figure difficult to interpret when only the discrete frequency points are plotted.

From Fig. 7, it can be seen that as the amplitude of the test signal decreases, then the estimate becomes biased toward $-1 / C$. The bias was estimated using the result for the asymptotic symmetrical distribution. The plot shows that this bias estimate predicts the behavior of the bias to a high degree of accuracy for our estimate $\hat{G}_{a v}$ under the test conditions.

It can also be seen from Fig. 7 that the perceived variance around the mean appears to approach zero when the SNR is either low or high. This accords with the observations made in Section VIII-A. Indeed, one can readily estimate the variance using Theorem 15 and (98). The plot of the standard deviation around the bias is then as shown in Fig. 8. Note that the standard deviation does indeed capture the correct qualitative and quantitative features of the experimental results.

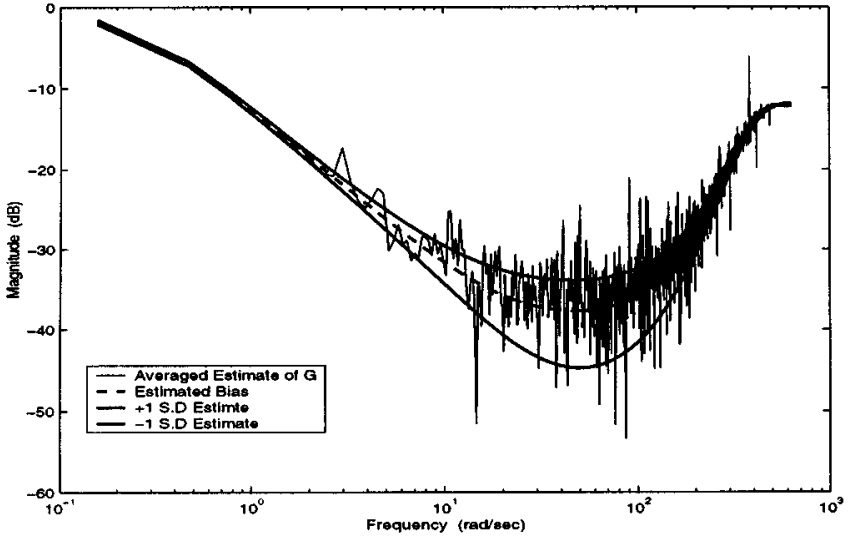

Fig. 8. Estimate of \pm 1 standard deviation around the bias.

\section{CONCLUSION}

The key result of this paper has been to rigorously quantify the finite sample properties of the indirect nonparametric estimator of the frequency response of a linear system operating in closed-loop assuming Gaussian noise. The key to doing this has been to utilize regularization in the form of an exclusion zone. We have shown that, with an exclusion zone, the estimate of $G$ under closed-loop conditions is biased for finite samples but asymptotically unbiased when a periodic signal is applied to the reference. Furthermore, the transition between an unbiased and a biased estimate occurs rapidly allowing easy distinction between the estimates. Similarly, an exclusion zone leads to a result for the variance which holds generally for arbitrary SNR. This result is consistent with earlier results for the high SNR case. Our results hold generally but, inter-alia, show that the previously published asymptotic results are valid only in the limit as the variance becomes vanishingly small. We have also shown that our results are relatively insensitive to a range of exclusion zone diameters.

\section{APPENDIX}

A. Proof of Lemma 10: The probability of lying inside the exclusion zone is

$$
p 1=\int_{A} \frac{1}{2 \pi \sigma_{s c}^{2}} e^{-|z|^{2} / 2 \sigma_{s c}^{2}} d z \text {. }
$$

Shifting the origin to the left by 1 and defining a new variable, $z=\zeta+1$, gives

$$
p 1=\int_{A} \frac{1}{2 \pi \sigma_{s c}^{2}} \exp \left(\frac{-|\zeta+1|^{2}}{2 \sigma_{s c}^{2}}\right) d \zeta .
$$

Changing to polar coordinates, we have

$$
\begin{aligned}
p 1=\int_{0}^{\epsilon_{1}} \frac{r}{2 \pi \sigma_{s c}^{2}} \exp \left(\frac{-\left(r^{2}+1\right)}{2 \sigma_{s c}^{2}}\right) & \\
& \int_{0}^{2 \pi} \cdot \exp \left(\frac{-r \cos \phi}{\sigma_{s c}^{2}}\right) d \phi d r .
\end{aligned}
$$

The integral with respect to $\phi$ can be expressed as a Bessel function of the first kind [23]

$$
J_{0}\left(\frac{j r}{\sigma_{s c}^{2}}\right)=\sum_{k=0}^{\infty} \frac{(-1)^{k}}{(k !)^{2}}\left(\frac{j r}{2 \sigma_{s c}^{2}}\right)^{2 k} \text {. }
$$


Substituting (102) into (101) and changing the order of integration and summation, we obtain

$$
\begin{aligned}
p 1=\frac{1}{\sigma_{s c}^{2}} \sum_{k=0}^{\infty} & \frac{(-1)^{k}}{(k !)^{2}}\left(\frac{j}{2 \sigma_{s c}^{2}}\right)^{2 k} \\
& \int_{0}^{\epsilon_{1}} r^{2 k+1} \cdot \exp \left(\frac{-\left(r^{2}+1\right)}{2 \sigma_{s c}^{2}}\right) d r .
\end{aligned}
$$

By substituting $\tau=r^{2} / 2 \sigma_{s c}^{2}$ in (103), we obtain

$$
p 1=e^{-1 / 2 \sigma_{s c}^{2}} \sum_{k=0}^{\infty} \frac{1}{(k !)^{2} 2^{k} \sigma_{s c}^{2 k}} \int_{0}^{\epsilon_{1}^{2} / 2 \sigma_{s c}^{2}} \tau^{k} e^{-\tau} d \tau .
$$

Expressing (104) as an incomplete gamma function

$$
p 1=e^{-1 / 2 \sigma_{s c}^{2}} \sum_{k=0}^{\infty} \frac{1}{(k !)^{2} 2^{k} \sigma_{s c}^{2 k}} \gamma\left(k+1, \frac{\epsilon_{1}^{2}}{2 \sigma_{s c}^{2}}\right) .
$$

Thus the normalization constant is given by $c_{h}=1-p_{1}$ which then yields the result.

B. Proof of Theorem 12: Part 1) With reference to Fig. 1, the estimation error can be expressed as

$$
\tilde{G}=\hat{G}-G_{o}=\frac{\tilde{T}}{C\left(1-T_{o}\right)\left(1-T_{o}-\tilde{T}\right)} .
$$

The expected value, in the case of the symmetric asymptotic distribution including an exclusion zone, is given by

$\mathbf{E}\{\tilde{G}\}=\frac{1}{C S_{o}} \iint_{\mathbb{C}-A} \frac{\frac{\tilde{T}}{S_{o}}}{\left(1-\frac{\tilde{T}}{S_{o}}\right)} \bar{p}(R, \lambda: \sigma) d \lambda d R$

where $\mathbb{C}$ denotes the entire complex plane and $A$ is the excluded region, shown in Fig. 3, of radius $\epsilon_{1}$.

We change to normalized variables using $\operatorname{Re}^{j \lambda}=\tilde{T} / S_{o}$. The areas to be integrated over are shown in Fig. 3. The scaled constants defining the exclusion zone become

$$
\epsilon_{1}=\frac{\epsilon}{\left|S_{o}\right|} \quad \text { and } \quad \epsilon_{2}=\sin ^{-1} \frac{\epsilon}{\left|S_{o}\right|}
$$

where $\epsilon$ is as in Fig. 2.

Hence, the expected value, in the case of the asymmetric asymptotic distribution, becomes

$$
\begin{aligned}
\mathbf{E}\left\{\tilde{G}_{h}\right\} & =\frac{1}{C S_{o}} \iint_{\mathbb{C}-A} \frac{\operatorname{Re}^{j \lambda}}{\left(1-\mathrm{Re}^{j \lambda}\right)} \bar{p}\left(R, \lambda: \sigma_{s c}\right) d \lambda d R \\
& =\frac{-1}{C S_{o}}+\iint_{\mathbb{C}-A} f_{b}(R, \lambda) d \lambda d R
\end{aligned}
$$

where

$$
f_{b}(R, \lambda)=\frac{\bar{p}\left(R, \lambda: \sigma_{s c}\right)}{C S_{o}\left(1-R e^{j \lambda}\right)} .
$$

The normalized probability density function is given by

$$
\begin{aligned}
& \bar{p}\left(R, \lambda: \sigma_{s c}\right) \\
& \quad= \begin{cases}\frac{R}{c_{h} 2 \pi \sigma_{s c}^{2}} e^{-R^{2} / 2 \sigma_{s c}^{2}} & \text { for }(R, \lambda) \in(\mathbb{C}-A) \\
0 & \text { for }(R, \lambda) \in A\end{cases}
\end{aligned}
$$

where $c_{h}$, is defined in (60). To simplify the integration, we divide the integral into four regions

$$
\mathbf{E}\left\{\tilde{G}_{h}\right\}=\frac{-1}{C S_{o}}+I_{1}+I_{2}+I_{3}+I_{4}
$$

where

$$
I_{k}=\iint_{A_{k}} f_{b}(R, \lambda) d \lambda d R
$$

and the integration areas are

$$
\begin{aligned}
& A_{1}=\left\{R, \lambda: 0 \leq R<1-\epsilon_{1}, 0 \leq \lambda<2 \pi\right\} \\
& A_{2}=\left\{R, \lambda: 1+\epsilon_{1} \leq R<\infty, 0 \leq \lambda<2 \pi\right\} \\
& A_{3}=\left\{R, \lambda: 1-\epsilon_{1} \leq R<1+\epsilon_{1}, \epsilon_{2} \leq \lambda<2 \pi-\epsilon_{2}\right\}
\end{aligned}
$$

$$
A_{4}=\{R, \lambda \text { : Area "B" in Fig. } 3\} .
$$

Integrating $I_{1}$ and $I_{2}$ in (113) we obtain the result of Theorem 12 Part 1).

Part 2) For $I_{3}$ in (113), consider the annulus, as shown in Fig. 3, excluding the areas A and B. The integration we need to perform is then

$$
I_{3}=\int_{1-\epsilon_{1}}^{1+\epsilon_{1}} \int_{\epsilon_{2}}^{2 \pi-\epsilon_{2}} f_{b}(R, \lambda) d \lambda d R .
$$

The factor $f_{b}(R, \lambda)$ in $(119)$ has a denominator containing the vector $1-\operatorname{Re}^{j \lambda}$ which is the length from " 1 " to any point within the annulus. We will next establish bounds by taking the smallest length vector around the annulus for any given angle. Fig. 9 shows the annulus divided intofive regions to simplify the integration. Consider

$$
I_{3}=I_{31}+I_{32}+I_{33}+I_{34}+I_{35}
$$

where

$$
I_{3 k}=\iint_{A_{3 k}} f_{b}(R, \lambda) d \lambda d R .
$$

To explain the regions $A_{3 k}$ consider Fig. 9. For integration over Region 1 , we define by $\beta$ the angle that gives the shortest length vector $(r)$ to any point on a line sweeping from an angle of $\epsilon_{2}$ to a maximum angle. The shortest length vector, to this line, occurs when the angle of intersection is $90^{\circ}$. The maximum angle to be integrated over for this region occurs when the vector also intersects the inner ring of the annulus. The angle is then calculated by basic trigonometry and is found to be $\cos ^{-1}\left(1-\epsilon_{1}\right)$.

Region 5 will be of the same size as Region 1 due to symmetry. Hence, we can establish the integration limits

$$
\begin{aligned}
& A_{31}=\left\{R, \lambda: 1-\epsilon_{1} \leq R<1+\epsilon_{1},\right. \\
& \left.\epsilon_{2} \leq \lambda<\cos ^{-1}\left(1-\epsilon_{1}\right)\right\} \\
& A_{32}=\left\{R, \lambda: 1-\epsilon_{1} \leq R<1+\epsilon_{1}\right. \\
& \left.\cos ^{-1}\left(1-\epsilon_{1}\right) \leq \lambda<\frac{\pi}{2}\right\} \\
& A_{33}=\left\{R, \lambda: 1-\epsilon_{1} \leq R<1+\epsilon_{1}, \frac{\pi}{2} \leq \lambda<\frac{3 \pi}{2}\right\} \\
& A_{34}=\left\{R, \lambda: 1-\epsilon_{1} \leq R<1+\epsilon_{1},\right. \\
& \left.\frac{3 \pi}{2} \leq \lambda<2 \pi-\cos ^{-1}\left(1-\epsilon_{1}\right)\right\} \\
& A_{35}=\left\{R, \lambda: 1-\epsilon_{1} \leq R<1+\epsilon_{1}\right. \text {, } \\
& \left.2 \pi-\cos ^{-1}\left(1-\epsilon_{1}\right) \leq \lambda<2 \pi-\epsilon_{2}\right\} .
\end{aligned}
$$




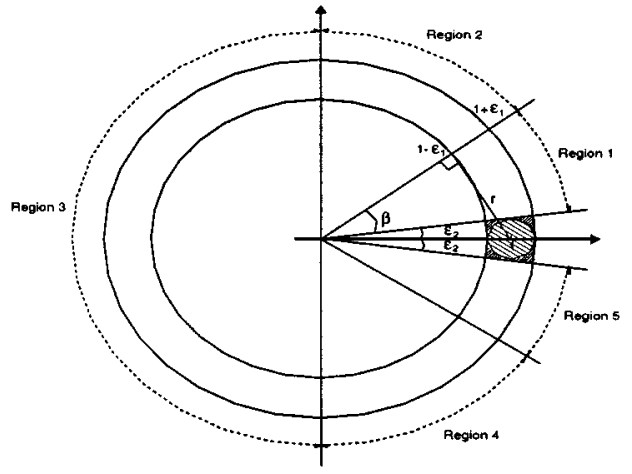

Fig. 9. Integration regions.

Next, we establish the length of the vector as a function of the angle. For region 1, using simple trigonometry we find the vector length $(r)$ to be $r=\sin (\lambda)$.

The integral for region 1 is thus

$$
I_{31}=\frac{1}{C S_{o}} \iint_{A_{31}} \frac{1}{\left(1-\operatorname{Re}^{j \lambda}\right)} \bar{p}\left(R, \lambda: \sigma_{s c}\right) d \lambda d R .
$$

Integrating an upperbound on $I_{31}$ with respect to angle

$$
\begin{aligned}
\int_{\epsilon_{2}}^{\cos ^{-1}\left(1-\epsilon_{1}\right)} \frac{1}{\left|1-\operatorname{Re}^{j \lambda}\right|} d \lambda= & \int_{\epsilon_{2}}^{\cos ^{-1}\left(1-\epsilon_{1}\right)} \frac{1}{\sin (\lambda)} d \lambda \\
= & \log \tan \left(\frac{\cos ^{-1}\left(1-\epsilon_{1}\right)}{2}\right) \\
& -\log \tan \left(\frac{\epsilon_{2}}{2}\right)
\end{aligned}
$$

Substituting (128) into (127) and integrating

$$
\begin{aligned}
I_{31} \leq & \frac{1}{2 \pi c_{h} C S_{o}}\left[\log \tan \left(\frac{\cos ^{-1}\left(1-\epsilon_{1}\right)}{2}\right)-\log \tan \left(\frac{\epsilon_{2}}{2}\right)\right] \\
& \cdot\left[\exp \left(\frac{-\left(1-\epsilon_{1}\right)^{2}}{2 \sigma_{s c}^{2}}\right)-\exp \left(\frac{-\left(1+\epsilon_{1}\right)^{2}}{2 \sigma_{s c}^{2}}\right)\right] \cdot
\end{aligned}
$$

Overbounding the integrals $I_{32}$ and $I_{33}$ we obtain

$$
\begin{aligned}
I_{32} \leq & \frac{1}{2 \pi c_{h} \sigma_{s c}^{2} C S_{o} \sqrt{2 \epsilon_{1}-\epsilon_{1}^{2}}} \iint_{A_{32}} \operatorname{Re}^{-R^{2} / 2 \sigma_{s c}^{2}} d \lambda d R \\
= & \frac{\frac{\pi}{2}-\cos ^{-1}\left(1-\epsilon_{1}\right)}{2 \pi c_{h} C S_{o} \sqrt{2 \epsilon_{1}-\epsilon_{1}^{2}}} \\
I_{33} \leq & \frac{\cdot\left(\exp \left(\frac{-\left(1-\epsilon_{1}\right)^{2}}{2 \sigma_{s c}^{2}}\right)-\exp \left(\frac{-\left(1+\epsilon_{1}\right)^{2}}{2 \sigma_{s c}^{2}}\right)\right)}{2 \pi c_{h} \sigma_{s c}^{2} C S_{o} \sqrt{2-2 \epsilon_{1}+\epsilon_{1}^{2}}} \iint_{A_{33}} \operatorname{Re}^{-R^{2} / 2 \sigma_{s c}^{2}} d \lambda d R \\
= & \frac{\pi}{2 \pi c_{h} C S_{o} \sqrt{2-2 \epsilon_{1}+\epsilon_{1}^{2}}} \\
& \cdot\left(\exp \left(\frac{-\left(1-\epsilon_{1}\right)^{2}}{2 \sigma_{s c}^{2}}\right)-\exp \left(\frac{-\left(1+\epsilon_{1}\right)^{2}}{2 \sigma_{s c}^{2}}\right)\right) .
\end{aligned}
$$

Regions 1 and 5, 2 and 4 are symmetric hence, $I_{31}=I_{35}$ and $I_{32}=I_{34}$. Substituting (129), (131), and (133) into (120) and taking into account symmetry we obtain an upper bound for (119), which in terms of our result is $K_{b 1}$.

We next proceed to $K_{b 2}$. We will overbound the integral $I_{4}$ in (113), which represents area "B" in Fig. 3

$$
I_{4}=\iint_{B} f_{b}(R, \lambda) d \lambda d R .
$$

We further divide area " $\mathrm{B}$ " into smaller regions $\mathrm{B} 1$ and $\mathrm{B} 2$, where $\mathrm{B} 1$ is the shaded region of " $\mathrm{B}$ " that lies within radius one, and $\mathrm{B} 2$ is the area lying outside radius one. Thus we partition $I_{4}$, with respect to $\mathrm{B} 1$ and $\mathrm{B} 2$, as

$$
I_{4}=I_{41}+I_{42} \text {. }
$$

We then overbound the integrals $I_{41}$ and $I_{42}$ as follows:

$$
\begin{aligned}
I_{41} & \leq \frac{1}{2 \pi c_{h} \sigma_{s c}^{2} C S_{o} \epsilon_{1}} \exp \left(\frac{-\left(1-\epsilon_{1}\right)^{2}}{2 \sigma_{s c}^{2}}\right) \iint_{B 1} d \lambda d R \\
& =\frac{\left(2 \epsilon_{2}-\epsilon_{1}\left(\epsilon_{2}-\frac{\pi}{2}\right)\right)}{2 \pi c_{h} \sigma_{s c}^{2} C S_{o}} \exp \left(\frac{-\left(1-\epsilon_{1}\right)}{2 \sigma_{s c}^{2}}\right) \\
I_{42} & \leq \frac{\left(1+\epsilon_{1}\right)}{2 \pi c_{h} \sigma_{s c}^{2} C S_{o} \epsilon_{1}} \exp \left(\frac{-1}{2 \sigma_{s c}^{2}}\right) \iint_{B 2} d \lambda d R \\
& =\frac{\left(1+\epsilon_{1}\right)\left(2 \epsilon_{2}+\epsilon_{1}\left(\epsilon_{2}-\frac{\pi}{2}\right)\right)}{2 \pi c_{h} \sigma_{s c}^{2} C S_{o}} \exp \left(\frac{-1}{2 \sigma_{s c}^{2}}\right)
\end{aligned}
$$

Substituting (137) and (139) into (135) we obtain an upper bound on (134), which in terms of our result, is $K_{b 2}$.

C. Proof of Theorem 13: The true bias in the estimation error is given by

$$
\mathbf{E}\{\tilde{G}\}=\frac{-1}{C S_{o}}+\frac{1}{C S_{o}} \iint_{\mathbb{C}-A} \frac{\bar{p}_{T}(R, \lambda)}{\left(1-\operatorname{Re}^{j \lambda}\right)} d \lambda d R .
$$

For the asymptotic symmetrical distribution, the bias is given by (110). The magnitude of the difference between (140) and (110), is

$$
\begin{aligned}
\left|\mathbf{E}\{\tilde{G}\}-\mathbf{E}\left\{\tilde{G}_{h}\right\}\right| \leq \frac{1}{|C|\left|S_{o}\right|} \\
\iiint_{\mathbb{C}-A} \cdot \frac{\left|\bar{p}_{T}(R, \lambda)-\bar{p}\left(R, \lambda: \sigma_{s c}\right)\right|}{\left|1-\operatorname{Re}^{j \lambda}\right|} d \lambda d R .
\end{aligned}
$$

As the true distribution of $\tilde{T}$ is of an elliptical shape, the above integration is difficult. Thus to simplify, we bound the difference between $\bar{p}_{T}(R, \lambda)$ and $\bar{p}\left(R, \lambda: \sigma_{s c}\right)$ by the difference between $\bar{p}\left(R, \lambda: \sigma_{s c \max }\right)$ and $\bar{p}\left(R, \lambda: \sigma_{s c \min }\right)$

$$
\left|\mathbf{E}\{\tilde{G}\}-\mathbf{E}\left\{\tilde{G}_{h}\right\}\right| \leq K_{e} \iint_{\mathbb{C}-A} \frac{P_{A}}{\left|1-\operatorname{Re}^{j \lambda}\right|} d \lambda d R
$$

where

$$
\begin{aligned}
& K_{e}=\frac{1}{|C|\left|S_{o}\right|} \\
& P_{A}=k_{s c \max } \bar{p}\left(R, \lambda: \sigma_{s c \max }\right)-k_{s c \min } \bar{p}\left(R, \lambda: \sigma_{s c \min }\right)
\end{aligned}
$$


Next, we partition the integral into four regions

$$
\left|\mathbf{E}\{\tilde{G}\}-\mathbf{E}\left\{\tilde{G}_{h}\right\}\right| \leq I_{1}+I_{2}+I_{3}+I_{4}
$$

where

$$
\begin{array}{r}
I_{k}=\iint_{A_{k}} f_{e}(R, \lambda) d \lambda d R \\
f_{e}(R, \lambda)=\frac{P_{A}}{|C|\left|S_{o}\right|\left|1-\mathrm{Re}^{j \lambda}\right|}
\end{array}
$$

and limits $A_{k}$ are defined in (115)-(118). Integrating $I_{1}$

$$
\begin{aligned}
I_{1} & =K_{e} \int_{0}^{1-\epsilon_{1}} \int_{0}^{2 \pi} \frac{P_{A}}{\left|1-\operatorname{Re}^{j \lambda}\right|} d \lambda d R \\
& \leq K_{e} \int_{0}^{1-\epsilon_{1}} \frac{P_{B}}{1-R} d R \\
& =K_{e} \int_{0}^{1-\epsilon_{1}}\left(1+R+R^{2}+\cdots\right) P_{B} d R
\end{aligned}
$$

where

$$
P_{B}=k_{s c \max } \bar{p}_{\max }(R)-k_{s c \min } \bar{p}_{\min }(R)
$$

and $\bar{p}_{\text {min }}(R)$ and $\bar{p}_{\text {max }}(R)$ are as in (73) and (74), $k_{s c \text { min }}$, and $k_{s c \max }$ are as in (58) and (59).

Similarly, we integrate $I_{2}$

$$
\begin{aligned}
I_{2} & =K_{e} \int_{1+\epsilon_{1}}^{\infty} \int_{0}^{2 \pi} \frac{P_{A}}{\mid 1-\mathrm{Re}^{j \lambda \mid}} d \lambda d R \\
& \leq K_{e} \int_{1+\epsilon_{1}}^{\infty} \frac{-1}{1-R} P_{B} d R \\
& =K_{e} \int_{1+\epsilon_{1}}^{\infty}\left(\frac{1}{R}+\frac{1}{R^{2}}+\frac{1}{R^{3}}+\cdots\right) P_{B} d R .
\end{aligned}
$$

Finally, we bound the constants $K_{e 1}$ and $K_{e 2}$. First, we integrate $I_{3}$ in (145)

$$
I_{3}=\int_{1-\epsilon_{1}}^{1+\epsilon_{1}} \int_{\epsilon_{2}}^{2 \pi-\epsilon_{2}} f_{e}(R, \lambda) d \lambda d R
$$

where $f_{e}(R, \lambda)$ is as in (147). We then integrate, as in Theorem $12,(120)$, where

$$
I_{3 k}=\iint_{A_{3 k}} f_{e}(R, \lambda) d \lambda d R
$$

and $A_{3 k}$ is given in (122)-(126). Using previous results from Theorem 12, we now integrate in each region

$$
\begin{aligned}
I_{31}= & K_{e} \int_{1-\epsilon_{1}}^{1+\epsilon_{1}} \int_{\epsilon_{2}}^{\cos ^{-1}\left(1-\epsilon_{1}\right)} \frac{P_{A}}{\mid 1-\operatorname{Re}^{j \lambda \mid}} d R \\
= & \frac{K_{e}}{2 \pi}\left[\log \tan \frac{\lambda}{2}\right]_{\epsilon_{2}}^{\cos ^{-1}\left(1-\epsilon_{1}\right)} \int_{1-\epsilon_{1}}^{1+\epsilon_{1}} P_{B} d R \\
= & \frac{K_{e}}{2 \pi}\left[\log \tan \frac{\lambda}{2}\right]_{\epsilon_{2}}^{\cos ^{-1}\left(1-\epsilon_{1}\right)} \\
& \cdot\left[\frac{k_{s c \max }}{c_{\max }}\left(\exp \left(\frac{-\left(1-\epsilon_{1}\right)^{2}}{2 \sigma_{s c \max }^{2}}\right)-\exp \left(\frac{-\left(1+\epsilon_{1}\right)^{2}}{2 \sigma_{s c \max }^{2}}\right)\right)\right. \\
& \left.-\frac{k_{s c \min }}{c_{\min }}\left(\exp \left(\frac{-\left(1-\epsilon_{1}\right)^{2}}{2 \sigma_{s c \min }^{2}}\right)-\exp \left(\frac{-\left(1+\epsilon_{1}\right)^{2}}{2 \sigma_{s c \min }^{2}}\right)\right)\right] .
\end{aligned}
$$

Bounding the integral $I_{32}$, we obtain

$$
\begin{aligned}
I_{32} \leq & \frac{K_{e}}{\sqrt{2 \epsilon_{1}-\epsilon_{1}^{2}}} \int_{1-\epsilon_{1}}^{1+\epsilon_{1}} \int_{\cos ^{-1}\left(1-\epsilon_{1}\right)}^{\pi / 2} P_{A} d \lambda d R \\
= & \frac{K_{e}\left(\frac{\pi}{2}-\cos ^{-1}\left(1-\epsilon_{1}\right)\right)}{2 \pi \sqrt{2 \epsilon_{1}-\epsilon_{1}^{2}}} \\
& \cdot\left[\frac{k_{s c \max }}{c_{\max }}\left(\exp \left(\frac{-\left(1-\epsilon_{1}\right)^{2}}{2 \sigma_{s c \max }^{2}}\right)-\exp \left(\frac{-\left(1+\epsilon_{1}\right)^{2}}{2 \sigma_{s c \max }^{2}}\right)\right)\right. \\
& \left.-\frac{k_{s c \min }}{c_{\min }}\left(\exp \left(\frac{-\left(1-\epsilon_{1}\right)^{2}}{2 \sigma_{s c \min }^{2}}\right)-\exp \left(\frac{-\left(1+\epsilon_{1}\right)^{2}}{2 \sigma_{s c \min }^{2}}\right)\right)\right]
\end{aligned}
$$

For $I_{33}$, we obtain

$$
\begin{aligned}
I_{33} \leq & \frac{K_{e}}{\sqrt{2-2 \epsilon_{1}+\epsilon_{1}^{2}}} \int_{1-\epsilon_{1}}^{1+\epsilon_{1}} \int_{\pi / 2}^{3 \pi / 2} P_{A} d \lambda d R \\
= & \frac{K_{e}}{2 \sqrt{2-2 \epsilon_{1}+\epsilon_{1}^{2}}} \\
& \cdot\left[\frac{k_{s c \max }}{c_{\max }}\left(\exp \left(\frac{-\left(1-\epsilon_{1}\right)^{2}}{2 \sigma_{s c \max }^{2}}\right)-\exp \left(\frac{-\left(1+\epsilon_{1}\right)^{2}}{2 \sigma_{s c \max }^{2}}\right)\right)\right. \\
& \left.-\frac{k_{s c \min }}{c_{\min }}\left(\exp \left(\frac{-\left(1-\epsilon_{1}\right)^{2}}{2 \sigma_{s c \min }^{2}}\right)-\exp \left(\frac{-\left(1+\epsilon_{1}\right)^{2}}{2 \sigma_{s c \min }^{2}}\right)\right)\right] .
\end{aligned}
$$

Substituting (159), (161), and (163) into (120) and taking into account symmetry, we obtain an upper bound for (155), which in terms of our result, is $K_{e 1}$.

Next, we bound the integral $I_{4}$ which is given by

$$
I_{4}=\iint_{B} f_{e}(R, \lambda) d \lambda d R
$$

Again, we do this as in the proof of Theorem 12, (135). Thus, we bound the integrals $I_{41}$ and $I_{42}$ as follows:

$$
\begin{aligned}
I_{41} \leq & \frac{K_{e}}{2 \pi \epsilon_{1} \sqrt{\operatorname{det}(D)}}\left(\frac{1}{c_{\max }} \exp \left(\frac{-\left(1-\epsilon_{1}\right)^{2}}{2 \sigma_{s c \max }^{2}}\right)\right. \\
& \left.-\frac{1}{c_{\min }} \exp \left(\frac{-1}{2 \sigma_{s c \min }^{2}}\right)\right) \iint_{B 1} d \lambda d R \\
= & \frac{K_{e}}{2 \pi \sqrt{\operatorname{det}(D)}}\left(2 \epsilon_{2}-\epsilon_{1}\left(\epsilon_{2}-\frac{\pi}{2}\right)\right) \\
& \cdot\left(\frac{1}{c_{\max }} \exp \left(\frac{-\left(1-\epsilon_{1}\right)^{2}}{2 \sigma_{s c \max }^{2}}\right)-\frac{1}{c_{\min }} \exp \left(\frac{-1}{2 \sigma_{s c \min }^{2}}\right)\right) \\
I_{42} \leq & \frac{K_{e}\left(1+\epsilon_{1}\right)}{2 \pi \epsilon_{1} \sqrt{\operatorname{det}(D)}}\left(\frac{1}{c_{\max }} \exp \left(\frac{-1}{2 \sigma_{s c \max }^{2}}\right)\right. \\
& \left.-\frac{1}{c_{\min }} \exp \left(\frac{-\left(1+\epsilon_{1}\right)^{2}}{2 \sigma_{s c \min }^{2}}\right)\right) \iint_{B 2} d \lambda d R \\
= & \frac{K_{e}}{2 \pi \sqrt{\operatorname{det}(D)}}\left(1+\epsilon_{1}\right)\left(2 \epsilon_{2}+\epsilon_{1}\left(\epsilon_{2}-\frac{\pi}{2}\right)\right) \\
& \cdot\left(\frac{1}{c_{\max }} \exp \left(\frac{-1}{2 \sigma_{s c \max }^{2}}\right)-\frac{1}{c_{\min }} \exp \left(\frac{-\left(1+\epsilon_{1}\right)^{2}}{2 \sigma_{s c \min }^{2}}\right)\right) .
\end{aligned}
$$


Substituting (166) and (168) into (135), we obtain our result where $K_{e 2}=I_{4}$.

D. Proof of Corollary 14: Part 1) We first note that, in view of Theorem $12, \mathbf{E}\{\tilde{G}\}$ rapidly approaches $\mathbf{E}\left\{\tilde{G}_{h}\right\}$. We thus begin with $\tilde{G}_{h}$ as in (63). Now we check the probability density renormalization constant for convergence to 1, i.e., $\lim _{N \rightarrow \infty}\left|c_{h}\right|=1$. Substituting (54) into (60), we have

$$
c_{h}=1-e^{-N Z / 2} \sum_{k=0}^{\infty} \frac{(N Z)^{k}}{(k !)^{2} 2^{k}} \gamma\left(k+1, \frac{N Z \epsilon_{1}^{2}}{2}\right)
$$

where

$$
Z=\frac{\Delta A^{2}}{2 \mathcal{S}_{1}}
$$

The gamma function can be expressed as [23]

$$
\gamma\left(k+1, \frac{N Z \epsilon_{1}^{2}}{2}\right)=k !\left(1-e^{-N Z \epsilon_{1}^{2} / 2} \sum_{l=0}^{k} \frac{\left(N Z \epsilon_{1}^{2}\right)^{l}}{l ! 2^{l}}\right) .
$$

Hence

$$
c_{h}=1-p_{11} p_{12}
$$

where

$$
\begin{aligned}
& p_{11}=e^{-N Z / 2} \sum_{k=0}^{\infty} \frac{(N Z)^{k}}{k ! 2^{k}} \\
& p_{12}=1-e^{-N Z \epsilon_{1}^{2} / 2} \sum_{l=0}^{k} \frac{\left(N Z \epsilon_{1}^{2}\right)^{l}}{l ! 2^{l}} .
\end{aligned}
$$

Now, we see that $\lim _{N \rightarrow \infty} p_{11}=1$ and $\lim _{N \rightarrow \infty} p_{12}=0$. Hence, $\lim _{N \rightarrow \infty} c_{h}=1$.

Each term in (63) is evaluated separately. Considering $K_{b 1}$, we substitute (54) into (68) and express the result as

$$
\begin{aligned}
K_{b 1} \leq \frac{1}{\pi c_{h} C S_{O}} f\left(\epsilon_{1}, \epsilon_{2}\right) & \left(\exp \left(\frac{-N\left(1-\epsilon_{1}\right)^{2} Z}{2}\right)\right. \\
& \left.-\exp \left(\frac{-N\left(1+\epsilon_{1}\right)^{2} Z}{2}\right)\right)
\end{aligned}
$$

where $f\left(\epsilon_{1}, \epsilon_{2}\right)$ is the term in square brackets in (68).

Then it is easily seen, recalling $\lim _{N \rightarrow \infty}\left|c_{h}\right|=1$, that $\lim _{N \rightarrow \infty}\left(K_{b 1}\right)=0$. Similarly, from (69), $K_{b 2}$ can be expressed as

$$
\begin{aligned}
K_{b 2} \leq \frac{N Z}{\pi c_{1} C S_{o}}\left(f_{1}\left(\epsilon_{1}, \epsilon_{2}\right) e^{\left(-N\left(1-\epsilon_{1}\right)^{2} Z\right) / 2}\right. & \\
& \left.+f_{2}\left(\epsilon_{1}, \epsilon_{2}\right) e^{(-N Z) / 2}\right)
\end{aligned}
$$

where $f_{1}\left(\epsilon_{1}, \epsilon_{2}\right)$ and $f_{2}\left(\epsilon_{1}, \epsilon_{2}\right)$ are the terms in the square and curly brackets, respectively, in (69). Then $\lim _{N \rightarrow \infty}\left(K_{b 2}\right)=0$. The first term in (63) is trivial, namely

$$
\lim _{N \rightarrow \infty}\left(\frac{-1}{C S o}\right)=\frac{-1}{C S o} .
$$

Substituting (54) into the remaining term in (63) we have

$$
\lim _{N \rightarrow \infty}\left(\frac{1}{c_{h} C S_{o}}\left(1-e^{-K N}\right)\right)=\frac{1}{C S_{o}}
$$

where

$$
K=\frac{\Delta A_{i}^{2}}{4 \mathcal{S}_{1}\left(\omega_{i}\right)} .
$$

Then for any $\xi>0, \exists M$ s.t. for $N>M$ we have $|\mathbf{E}\{\tilde{G}\}|<\xi$. This establishes the result.

Part 2) Immediate from (63).

E. Proof of Theorem 15: Part 1) With reference to (106), the variance of the estimation error, with the exclusion zone is given by

$$
\mathbf{E}\left\{\tilde{G} \tilde{G}^{*}\right\}=\frac{1}{|C|^{2}\left|S_{o}\right|^{4}} \iint_{\mathbb{C}-A} \frac{|\tilde{T}|^{2} \bar{p}_{T}(R, \lambda)}{\left|1-\frac{\tilde{T}}{S_{o}}\right|^{2}} d \lambda d R .
$$

We will overbound the distribution $\bar{p}_{T}(R, \lambda)$ via the Rayleigh distribution as in (49). Thus we replace $\bar{p}_{T}(R, \lambda)$ by $k_{\max } \bar{p}\left(R, \lambda: \sigma_{\max }\right)$.

We also change to normalized quantities and define $\mathrm{Re}^{j \lambda}$ as $\tilde{T} /\left|S_{o}\right|$ and expand the integral to include the different regions. Hence, the variance of the error in the estimate $\hat{G}$ is given by

$$
\mathbf{E}\left\{\tilde{G} \tilde{G}^{*}\right\} \leq \iint_{\mathbb{C}-A} f_{v}(R, \lambda) d \lambda d R
$$

where

$$
f_{v}(R, \lambda)=\frac{R^{2} k_{s c \max } \bar{p}\left(R, \lambda: \sigma_{s c \max }\right)}{|C|^{2}\left|S_{o}\right|^{2}\left|1-\mathrm{Re}^{j \lambda}\right|^{2}} .
$$

As before, we divide the integral into regions as follows:

$$
I_{k}=\iint_{A_{k}} f_{v}(R, \lambda) d \lambda d R
$$

where $A_{k}$ is given in (115)-(118). Hence

$$
\mathbf{E}\left\{\tilde{G} \tilde{G}^{*}\right\} \leq I_{1}+I_{2}+I_{3}+I_{4} .
$$

Now consider the first term in (184)

$$
I_{1}=\frac{k_{s c \max }}{|C|^{2}\left|S_{o}\right|^{2}} \int_{0}^{1-\epsilon_{1}} \int_{0}^{2 \pi} \frac{R^{2} \bar{p}\left(R, \lambda: \sigma_{s c \max }\right)}{\left|1-\operatorname{Re}^{j \lambda}\right|^{2}} d \lambda d R .
$$

We first integrate with respect to $\lambda$, giving

$$
I_{1}=K_{v} \int_{0}^{1-\epsilon_{1}} \frac{R^{2}}{1-R^{2}} \bar{p}_{\max }(R) d R .
$$

Since $R<1$, we can express the integrand in terms of a convergent power series [24]

$$
\begin{aligned}
I_{1}=\frac{K_{v}}{c_{\max } \sigma_{s c \max }^{2}} \int_{0}^{1-\epsilon_{1}} R^{3}\left(1+R^{2}+R^{4}+\cdots\right) \\
\cdot \exp \left(\frac{-R^{2}}{2 \sigma_{s c \max }^{2}}\right) d R .
\end{aligned}
$$


Next, consider the second term in (184). Integrating with respect to $\lambda$, we obtain

$$
I_{2}=-K_{v} \int_{1+\epsilon_{1}}^{\infty} \frac{R^{2}}{1-R^{2}} \bar{p}_{\max }(R) d R .
$$

Here $R>1$, so we can also use a convergent power series for the integrand

$$
\begin{aligned}
& I_{2}=\frac{K_{v}}{c_{\max } \sigma_{s c \max }^{2}} \int_{1+\epsilon_{1}}^{\infty}(R+\left.\frac{1}{R}+\frac{1}{R^{3}}+\cdots\right) \\
& \cdot \exp \left(\frac{-R^{2}}{2 \sigma_{s c \max }^{2}}\right) d R .
\end{aligned}
$$

Substituting $I_{1}$ and $I_{2}$ in (184) with (187) and (189), respectively, also replacing the $I_{3}$ and $I_{4}$ terms with there integral expressions from (183) the result follows.

Part 2) Here, we closely overbound the constant terms $K_{v 1}$ and $K_{v 2}$. First, we consider the $I_{3}$ term in (184)

$$
I_{3}=\int_{1-\epsilon_{1}}^{1+\epsilon_{1}} \int_{\epsilon_{2}}^{2 \pi-\epsilon_{2}} f_{v}(R, \lambda) d \lambda d R .
$$

We integrate, as in Theorem 12, (120), where

$$
I_{3 k}=\iint_{A_{3 k}} f_{v}(R, \lambda) d \lambda d R .
$$

For region 1, the integral is

$$
\begin{aligned}
I_{31} & =K_{v} \iint_{A_{31}} \frac{R^{2} \bar{p}\left(R, \lambda: \sigma_{s c \max }\right)}{\left|1-\operatorname{Re}^{j \lambda}\right|^{2}} d \lambda d R \\
& =\frac{K_{v}}{2 \pi}\left(\cot \left(\epsilon_{2}\right)-\frac{1-\epsilon_{1}}{\sqrt{2 \epsilon_{1}-\epsilon_{1}^{2}}}\right) \int_{1-\epsilon_{1}}^{1+\epsilon_{1}} R^{2} \bar{p}_{\max }(R) d R \\
& =\frac{K_{v}}{2 \pi c_{\max }}\left(\cot \left(\epsilon_{2}\right)-\frac{1-\epsilon_{1}}{\sqrt{2 \epsilon_{1}-\epsilon_{1}^{2}}}\right) K_{3}
\end{aligned}
$$

where

$$
\begin{aligned}
K_{3}=2 \sigma_{s c \max }^{2} & {\left[\left(\frac{\left(1-\epsilon_{1}\right)^{2}}{2 \sigma_{s c \max }^{2}}+1\right) \exp \left(\frac{-\left(1-\epsilon_{1}\right)^{2}}{2 \sigma_{s c \max }^{2}}\right)\right.} \\
- & \left.\left(\frac{\left(1+\epsilon_{1}\right)^{2}}{2 \sigma_{s c \max }^{2}}+1\right) \exp \left(\frac{-\left(1+\epsilon_{1}\right)^{2}}{2 \sigma_{s c \max }^{2}}\right)\right] .
\end{aligned}
$$

Overbounding the integrals $I_{32}$ and $I_{33}$, we obtain

$$
\begin{aligned}
I_{32} & \leq \frac{K_{v}}{\epsilon_{1}\left(2-\epsilon_{1}\right)} \iint_{A_{32}} R^{2} \bar{p}\left(R, \lambda: \sigma_{s c \max }\right) d \lambda d R \\
& =\frac{K_{v}\left(\frac{\pi}{2}-\cos ^{-1}\left(1-\epsilon_{1}\right)\right)}{2 \pi \epsilon_{1}\left(2-\epsilon_{1}\right)} \int_{1-\epsilon_{1}}^{1+\epsilon_{1}} R^{2} \bar{p}_{\max }(R) d R \\
& =\frac{K_{v}\left(\frac{\pi}{2}-\cos ^{-1}\left(1-\epsilon_{1}\right)\right)}{2 \pi c_{\max } \epsilon_{1}\left(2-\epsilon_{1}\right)} K_{3} \\
I_{33} & \leq \frac{K_{v}}{\left(2-2 \epsilon_{1}+\epsilon_{1}^{2}\right)} \iint_{A_{33}} R^{2} \bar{p}\left(R, \lambda: \sigma_{s c \max }\right) d \lambda d R
\end{aligned}
$$

$$
\begin{aligned}
& =\frac{K_{v}}{2\left(2-2 \epsilon_{1}+\epsilon_{1}^{2}\right)} \int_{1-\epsilon_{1}}^{1+\epsilon_{1}} R^{2} \bar{p}_{\max }(R) d R \\
& =\frac{K_{v}}{2 c_{\max }\left(2-2 \epsilon_{1}+\epsilon_{1}^{2}\right)} K_{3} .
\end{aligned}
$$

Substituting (194), (198), and (201) into (120), and taking into account symmetry, we obtain an upper bound for $I_{3}$, which in terms of our result is expressed as $K_{v 1}$.

The integral $I_{4}$ in (184) is overbounded as follows:

$$
I_{4}=\iint_{B} f_{v}(R, \lambda) d \lambda d R .
$$

Again, we proceed as in the proof of Theorem 12, (135). Overbounding the integrals $I_{41}$ and $I_{42}$, we obtain

$$
\begin{aligned}
I_{41} & \leq \frac{K_{v}}{2 \pi c_{\max } \epsilon_{1}^{2} \sigma_{s c \max }^{2}} \exp \left(\frac{-\left(1-\epsilon_{1}\right)^{2}}{2 \sigma_{s c \max }^{2}}\right) \iint_{B 1} d \lambda d R \\
& =\frac{K_{v}\left(2 \epsilon_{2}-\epsilon_{1} \epsilon_{2}-\frac{\pi}{2} \epsilon_{1}\right)}{2 \pi c_{\max } \epsilon_{1} \sigma_{s c \max }^{2}} \exp \left(\frac{-\left(1-\epsilon_{1}\right)^{2}}{2 \sigma_{s c \max }^{2}}\right) \\
I_{42} & \leq \frac{K_{v}\left(1+\epsilon_{1}\right)^{3}}{2 \pi c_{\max } \epsilon_{1}^{2} \sigma_{s c \max }^{2}} \exp \left(\frac{-1}{2 \sigma_{s c \max }^{2}}\right) \iint_{B 2} d \lambda d R \\
& =\frac{K_{v}\left(2 \epsilon_{2}+\epsilon_{1} \epsilon_{2}-\frac{\pi}{2} \epsilon_{1}\right)\left(1+\epsilon_{1}\right)^{3}}{2 \pi c_{\max } \epsilon_{1} \sigma_{s c \max }^{2}} \exp \left(\frac{-1}{2 \sigma_{s c \max }^{2}}\right) .
\end{aligned}
$$

Substituting (204) and (206) into (135), we obtain an upper bound for (202), which in terms of our result is $K_{v 2}$.

F. Proof of Equation (93): We consider the terms in (83) individually. Expressing the first term as in (83), substituting (54) for $\sigma_{s c \max }^{2}$ and (84) for $K_{v}$, we have

$$
I_{1}=\frac{1}{|C|^{2}\left|S_{o}\right|^{2} \sqrt{\operatorname{det}(D)} c_{\max }} \int_{0}^{1-\epsilon_{1}} \frac{R^{3} e^{-R^{2} K N / 2}}{1-R^{2}} d R
$$

where

$$
K=\frac{\Delta A_{i}^{2}}{2 \mathcal{S}_{1}\left(\omega_{i}\right)} .
$$

From (30), as $N \rightarrow \infty, N \sqrt{\operatorname{det}(D)} \rightarrow 2 \mathcal{S}_{1}\left(\omega_{i}\right) / \Delta A_{i}^{2}$. Hence

$$
I_{1}=\frac{K N}{|C|^{2}\left|S_{o}\right|^{2} c_{\max }} \int_{0}^{1-\epsilon_{1}} \frac{R^{3}}{1-R^{2}} e^{-R^{2} K N / 2} d R .
$$

To simplify the integration, we separate $I_{1}$ into two parts

$$
\begin{aligned}
I_{1} & =I_{11}+I_{12} \\
I_{11} & =\frac{K N}{|C|^{2}\left|S_{\circ}\right|^{2} c_{\max }} \int_{0}^{1-\epsilon_{1}} R^{3} e^{-R^{2} K N / 2} d R \\
I_{12} & =\frac{K N}{|C|^{2}\left|S_{\circ}\right|^{2} c_{\max }} \int_{0}^{1-\epsilon_{1}} \frac{R^{5}}{1-R^{2}} e^{-R^{2} K N / 2} d R .
\end{aligned}
$$

Integrating (211), we have

$$
\begin{aligned}
I_{11}=\frac{2}{|C|^{2}\left|S_{o}\right|^{2} c_{\max } K N}\left[1-\exp \left(\frac{-K N\left(1-\epsilon_{1}\right)^{2}}{2}\right)\right. \\
\left.\cdot\left(1+\frac{K N\left(1-\epsilon_{1}\right)^{2}}{2}\right)\right] .
\end{aligned}
$$


For (212), we simplify further by overbounding the integral

$$
\begin{aligned}
I_{12} \leq & \frac{K N}{|C|^{2}\left|S_{o}\right|^{2} c_{\max } \epsilon_{1}} \int_{0}^{1-\epsilon_{1}} R^{\tilde{5}} \exp \left(\frac{-R^{2} K N}{2}\right) d R \\
= & \frac{4}{|C|^{2}\left|S_{o}\right|^{2} c_{\max } K^{2} N^{2} \epsilon_{1}}\left(2-\exp \left(\frac{-K N\left(1-\epsilon_{1}\right)^{2}}{2}\right)\right. \\
& \left.\cdot\left[2+K N\left(1-\epsilon_{1}\right)^{2}+\frac{K^{2} N^{2}\left(1-\epsilon_{1}\right)^{4}}{2}\right]\right)
\end{aligned}
$$

The second integral term in (83) under the aforementioned conditions becomes

$$
\begin{aligned}
I_{2} \leq & \frac{K N}{|C|^{2}\left|S_{o}\right|^{2} c_{\max } \epsilon_{1}} \int_{1+\epsilon_{1}}^{\infty} R^{3} \exp \left(\frac{-R^{2} K N}{2}\right) d R \\
= & \frac{2}{|C|^{2}\left|S_{o}\right|^{2} c_{\max } K N \epsilon_{1}} \exp \left(\frac{-K N\left(1+\epsilon_{1}\right)^{2}}{2}\right) \\
& \cdot\left(1+\frac{K N\left(1+\epsilon_{1}\right)^{2}}{2}\right)
\end{aligned}
$$

Overbounds for the constant terms $K_{v 1}$ and $K_{v 2}$ in (83) can be expressed, using the same procedure as follows:

$$
\begin{aligned}
K_{v 1} & \leq \frac{K_{v} \sigma_{s c \max }^{2}}{c_{\max }} f_{1}\left(\epsilon_{1}, \epsilon_{2}\right) \exp \left(\frac{-1}{2 \sigma_{s c \max }^{2}}\right) \\
& =\frac{1}{|C|^{2}\left|S_{o}\right|^{2} c_{\max }} f_{1}\left(\epsilon_{1}, \epsilon_{2}\right) e^{-K N / 2} \\
K_{v 2} & \leq \frac{K_{v}}{c_{\max }} f_{2}\left(\epsilon_{1}, \epsilon_{2}\right) \exp \left(\frac{-1}{2 \sigma_{s c \max }^{2}}\right) \\
& =\frac{K N}{|C|^{2}\left|S_{o}\right|^{2} c_{\max }} f_{2}\left(\epsilon_{1}, \epsilon_{2}\right) e^{-K N / 2}
\end{aligned}
$$

where $f_{1}\left(\epsilon_{1}, \epsilon_{2}\right)$ and $f_{2}\left(\epsilon_{1}, \epsilon_{2}\right)$ depend only on $\epsilon_{1}$ and $\epsilon_{2}$ and are constant with respect to $N$.

Combining (83), (213), (215), (217), (219), and (221), and taking the limit as the number of observations tends to infinity, it can then be seen that for any given $\xi>0, \exists M>0$, s.t. for $N>M\left|\mathbf{E}\left\{N \tilde{G} \tilde{G}^{*} / G G^{*}\right\}-\left(4 \mathcal{S}_{1}\left(\omega_{i}\right) /\left|T_{o}\right|^{2} \Delta A_{i}^{2}\right)\right|<\xi$. This establishes the result.

\section{ACKNOWLEDGMENT}

The authors would like to thank W. Heath for helpful comments on earlier versions of the paper.

\section{REFERENCES}

[1] L. Ljung, System Identification: Theory for the User, second ed. Upper Saddle River, NJ: Prentice-Hall, 1999.

[2] T. Söderström and P. Stoica, System Identification. Upper Saddle River, NJ: Prentice-Hall, 1989.

[3] P. E. Wellstead, "Non-parametric methods of system identification," $\mathrm{Au}$ tomatica, vol. 17, no. 1, pp. 55-69, 1981.
[4] - "Reference signals for closed-loop identification," Int. J. Control, vol. 26, no. 6, pp. 945-962, 1977.

[5] U. Forssell, "Closed-loop identification: Methods, theory and applications," Ph.D. dissertation, Linkoping Univ., Linkoping, Sweden, 1999.

[6] G. C. Goodwin and J. S. Welsh, "Analysis of a novel method of autotuning a multivariable plant based on quantization," in Proc. American Automatic Control Conf., San Diego, CA, June 1999, pp. 3347-3351.

[7] D. K. De Vries and P. M. J. Van den Hof, "Quantification of uncertainty in transfer function estimation," Automatica, vol. 31, pp. 3-11, 1982.

[8] P. M. T. Broersen, "A comparison of transfer function estimators," IEEE Trans. Instrum. Measure., vol. 44, pp. 657-661, June 1995.

[9] W. P. Heath, "Bias of indirect nonparametric transfer function estimates for plants in closed-loop," Automatica, vol. 37, no. 10, pp. 1529-1540, 2001.

[10] E. J. Hannan, Multiple Time Series. New York: Wiley, 1970.

[11] J. S. Bendat and A. G. Piersol, Engineering Applications of Correlation and Spectral Analysis. New York: Wiley, 1980.

[12] M. B. Priestley, Spectral Analysis and Time Series. New York: Academic, 1981.

[13] D. R. Brillinger and P. R. Krishnaiah, Handbook of Statistics 3: Time Series in the Frequency Domain. Amsterdam, The Netherlands: North Holland, 1983.

[14] K. R. Godfrey, "Correlation methods," Automatica, vol. 16, pp. 527-534, 1980.

[15] W. P. Heath, "Probability density function of indirect nonparametric transfer function estimates for plants in closed-loop," presented at the SYSID2000, Santa Barbara, CA, June 2000.

[16] — "Characterising nonparametric estimators in closed-loop: The finite data case," in Proc. Eur. Control Conf., Porto, Portugal, 2001, pp. 3558-3563.

[17] P. Guillaume, I. Kollar, and R. Pintelon, "Statistical analysis of nonparametric transfer function estimates," IEEE Trans. Instrum. Measure., vol. 45, pp. 594-600, Feb. 1996.

[18] P. M. T. Broersen, "On the statistical accuracy of the empirical transfer function estimator," in SYSID2000, Santa Barbara, CA, 2000.

[19] D. R. Brillinger, Time Series: Data Analysis and Theory. San Francisco, CA: Holden-Day, 1981.

[20] J. S. Welsh and G. C. Goodwin, "Background on open loop nonparametric estimation," Univ. Newcastle, CIDAC Tech. Rep. EE02044, 2002.

[21] T. W. Körner, Fourier Analysis. Cambridge, U.K.: Cambridge Univ. Press, 1988

[22] D. S. Bayard, "Statistical plant set estimation using Schroeder-phased mutlisinusoidal input design," J. Appl. Math. Comput., vol. 58, pp. 169-198, 1993.

[23] G. Arfken, Mathematical Methods for Physicists. New York: Academic, 1985.

[24] E. Kreyszig, Advanced Engineering Mathematics. New York: Wiley, 1979.

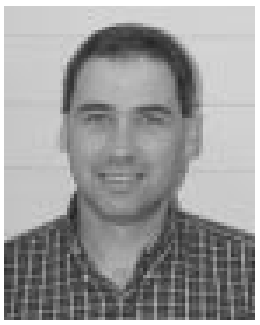

James S. Welsh was born in Maitland, Australia, in 1965. He received the B.E. degree (Hons. I) in electrical engineering from the University of Newcastle, Newcastle, Australia, in 1997 and is currently working toward the Ph.D. degree in electrical engineering at the same university.

From 1981 to 1997, he gained industrial experience with Bloomfield Collieries, Maitland, New South Wales, Australia, AES, Newcastle, New South Wales, Australia, and TYP Pty., Ltd., Newcastle, New South Wales, Australia. His research interests include autotuning, closed-loop identification, and process control. He has recently joined a research project on Powertrain Control with the Centre for Integrated Dynamics and Control at the University of Newcastle.

Mr. Welsh won the Institute of Engineers Australia Control Project Prize in 1997. 


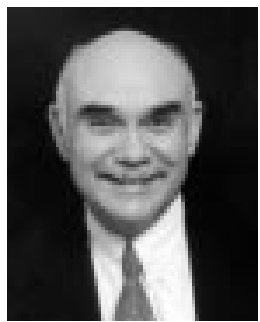

Graham C. Goodwin (M'74-SM'84-F'86) received the B.Sc. degree in physics, the B.E. degree in electrical engineering, and the Ph.D. degree, all from the University of New South Wales, New South Wales, Australia, in 1964, 1966, and 1970, respectively.

From 1970 to 1974 , he was a Lecturer in the Department of Computing and Control, Imperial College, London, U.K. Since 1974, he has been with the University of Newcastle, Australia, where he is currently Professor of Electrical Engineering and Commonwealth Government Federation Fellow. He is the coauthor of seven monographs, four edited volumes, and several technical papers.

Dr. Goodwin is the recipient of the USA Control Systems Society 1999 Hendrik Bode Lecture Prize, a Best Paper award by IEEE TRANSACTIONS ON AUTOMATIC CONTROL, and Best Engineering Text Book Award from the International Federation of Automatic Control. He is an Honorary Fellow of the Institute of Engineers, Australia, a Fellow of the Australian Academy of Science, a Fellow of the Australian Academy of Technology, Science, and Engineering, and a Member of the International Statistical Institute. 